\title{
Real-world evaluation of sodium-glucose co-transporter- 2 inhibitors and dipeptidyl peptidase-4 inhibitors for managing type 2 diabetes mellitus: a retrospective multi-ethnic cohort study
}

\author{
Louise Gek Huang Goh ${ }^{1}$. Jiandong Sun ${ }^{1}$ B Benjamin Shao Kiat Ong ${ }^{1} \cdot$ Daphne Khoo ${ }^{1}$ Chee Fang Sum ${ }^{2} \cdot \mathrm{Kwong} \mathrm{Ng}^{1}$
}

Received: 15 September 2021 / Accepted: 7 February 2022 / Published online: 3 March 2022

(c) The Author(s) 2022

\begin{abstract}
Purpose Sodium-glucose co-transporter-2 (SGLT2) inhibitors and dipeptidyl peptidase-4 (DPP4) inhibitors are increasingly used as second-line therapies in patients with type 2 diabetes. The aim of this study was to assess the real-world effects of SGLT2 inhibitors in a multi-ethnic population in Singapore.

Methods This retrospective cohort study examined patients diagnosed with and treated for diabetes from the Ministry of Health's administrative database. Differences in outcomes between treatment groups were assessed using Poisson regression. Demographics, clinical characteristics, previous diagnoses and hospitalisations, and diabetes medication history were used for propensity score matching. Subgroup analyses by ethnicity were performed. Effect size was estimated using risk ratios (RRs) with 95\% confidence intervals (CIs).

Results Patients initiating SGLT2 inhibitors were more likely to achieve glycaemic control target than DPP4 inhibitor-treated patients (RR 1.09; 95\% CI 1.04, 1.14). This was observed only in patients of Chinese ethnicity. A higher risk of diabetic ketoacidosis in SGLT2 inhibitor initiators was not observed. SGLT2 inhibitors were associated with reduced risk of hypoglycaemia (RR 0.69; 95\% CI 0.59, 0.82) and urinary tract infection (RR 0.52; 95\% CI 0.43, 0.63) but was not statistically significant for hypoglycaemia in Malay patients. Compared to DPP4 inhibitors, SGLT2 inhibitors were associated with 12\% and 34\% reduction in any-cause hospitalisation and all-cause mortality, respectively, potentially resulting in more than $\$ 50$ million savings over 10 years.

Conclusion SGLT2 inhibitors were associated with improvements in glycaemic control, reduced risk of complications, and was well tolerated. Ethnicity also plays a role and should be considered in future studies.
\end{abstract}

Keywords SGLT2 inhibitors $\cdot$ DPP4 inhibitors $\cdot$ type 2 diabetes $\cdot$ real-world $\cdot$ cohort study $\cdot$ diabetes outcomes

\section{Background}

Type 2 diabetes mellitus (T2DM) is a major concern worldwide and a main cause of death in most countries [1]. The International Diabetes Federation estimated that about 463 million adults had diabetes, with 4.2 million deaths due to diabetes in 2019 [2]. The Western Pacific region including

Kwong $\mathrm{Ng}$

ng_kwong_hoe@moh.gov.sg

1 Agency for Care Effectiveness (ACE), Ministry of Health, 16 College Road, College of Medicine Building,

Singapore 169854, Singapore

2 Diabetes Centre, Admiralty Medical Centre and Khoo Teck Puat Hospital, 90 Yishun Central, Singapore 768828, Singapore
Singapore had the highest number of deaths. In 2045, the number of people with diabetes is expected to increase to about 700 million. The prevalence of type 2 diabetes in Singapore adults aged 18 to 69 years will also double from $7.3 \%$ in 1990 to $15.0 \%$ in 2050 [3]. T2DM, if not well controlled, can further lead to complications like kidney failure, lower limb amputation, nerve damage, cardiovascular disease (CVD), loss of vision and severe disabilities [4-6]. In addition, Asian patients with T2DM tend to have an earlier onset compared to their Caucasian counterparts. Nearly one-fifth (18\%) were first diagnosed before 40 years old with a mean age of 32.9 years [7], compared to $13 \%$ in the United States (US) population aged between 18 to 44 years [8]. This further increases the risk of T2DM complications with longer disease duration. Optimal glycaemic control is thus crucial for preventing or delaying the development and 
progression of these complications [9]. A glycaemic control target, haemoglobin A1c (HbA1c) of below 7\% is considered reasonable for most adults to achieve and is used to identify patients with good control [10].

At present, the main classes of oral glucose-lowering agents registered in Singapore include biguanides, sulfonylureas, sodium-glucose co-transporter-2 (SGLT2) inhibitors, dipeptidyl peptidase-4 (DPP4) inhibitors, meglitinides, thiazolidinediones and alpha-glucosidase inhibitors [10]. Metformin, a biguanide, is recommended as first-line therapy due to its long-term efficacy and safety data [10]. It is well tolerated with a low risk of hypoglycaemia and weight gain [9]. However, it is often insufficient as a monotherapy to manage the condition as disease progresses, and multiple agents are required to control blood glucose [11]. While sulfonylureas are considered a suitable add-on therapy, they may increase the risk of hypoglycaemia. Newer drug classes like SGLT2 inhibitors and DPP4 inhibitors are increasingly being used as second-line hypoglycaemic agents when sulfonylureas are not tolerated or when hypoglycaemia is a concern $[12,13]$. Of note, DPP4 inhibitors can be used regardless of level of kidney function as long as the dosage is adjusted according to estimated glomerular filtration rate (eGFR) [14]. In contrast, SGLT2 inhibitors are contraindicated in those with moderate to severe kidney impairment $[15,16]$.

Three SGLT2 inhibitors (dapagliflozin, empagliflozin and canagliflozin) have been registered in Singapore since 2014. Their use is encouraged over DPP4 inhibitors given the availability of outcomes data and favourable cost-effectiveness [10, 17]. It remains unclear if the use of SGLT2 inhibitors in the local context is associated with the desired outcomes shown in clinical trials, while real-world studies comparing SGLT2 inhibitors with DPP4 inhibitors were mainly done in the western countries. To date, only a small local retrospective cohort study of 57 patients compared the effects of canagliflozin and sitagliptin on glycaemic control [18]. Given ethnicity is a significant predictor of $\mathrm{HbA} 1 \mathrm{c}$ levels, local evidence is needed to assess the real-world effectiveness of these newer drug classes in specific ethnic subgroups and the Singapore general population [19]. This national study aimed to compare the effects of SGLT2 inhibitors with DPP4 inhibitors on patient outcomes in an ethnically diverse Asian population using real-world evidence and further translate such differences into any potential healthcare cost savings.

\section{Methods}

\section{Study design and data source}

In this retrospective cohort study, the Ministry of Health $(\mathrm{MOH})$ 's administrative database containing national-level healthcare use data was accessed. It contained anonymised data from public hospitals and primary care clinics, with about 8 million diabetes prescription records up to 2018 . The study population was a large representative sample of patients with T2DM who sought treatment in the public healthcare setting in Singapore. Information on demographics, disease diagnoses, prescription records and investigation results of these patients were studied. Ethics approval was not required as the intent of this study was to assess the effect of SGLT2 inhibitors on clinical outcomes for the purpose of improving routine clinical care.

Disease diagnoses were recorded using the International Classification of Diseases, Tenth Revision Australian Modification (ICD-10 AM) codes. All T2DM patients aged 30 years and above receiving SGLT2 inhibitors (dapagliflozin, empagliflozin and canagliflozin) or DPP4 inhibitors (linagliptin, sitagliptin, vildagliptin and saxagliptin) at public healthcare institutions were included in the analyses. Patients were included in the study if they had a diagnosis and treatment for diabetes. For individuals with non-specific diagnosis codes, patients with type 1 diabetes mellitus (T1DM) were differentiated and excluded based on age at diagnosis and treatment. Patients diagnosed at age less than 40 years and on insulin only were classified as T1DM. This approach had also been used by other studies in identifying patients with T1DM [20, 21]. Non-residents who were not routinely managed and followed up in Singapore, and patients with no information on age, gender, age below 30 years or had a death record were also excluded.

\section{Patient selection and baseline characteristics}

T2DM patients newly initiating SGLT2 inhibitors or DPP4 inhibitors between January 2015 and December 2018 were included in this analysis. A washout period of one year was used to identify new users. The earliest prescription date was defined as the treatment initiation date. Patients were assigned to either SGLT2 inhibitor or DPP4 inhibitor-treated cohort dependent on the treatment they were initiated on. Those who had any prescriptions of studied drugs before the initiation date were excluded to restrict the cohorts to only new users. An intention to treat approach was used for the analysis where patients were followed from initiation of index treatment to observation of outcome or end of followup period (whichever was earlier).

Baseline characteristics were obtained for each patient during the one year before initiation. These variables included prescribing setting (public hospitals and primary care clinics), year of first prescription of SGLT2 inhibitors or DPP4 inhibitors, duration with diabetes, age, gender, ethnicity, resident status, body weight (in kilograms, $\mathrm{kg}$ ), blood pressure (in $\mathrm{mmHg}$ ), smoking status, subsidy status or socioeconomic status category, any hospitalisation, 
hospitalisation for DM complications [poor diabetes control (ICD-10 AM: E1*65), diabetic kidney complications $(\mathrm{E} 1 * 2 *)$, insulin resistance $(\mathrm{E} 1 * 72)$, hypoglycaemia $(\mathrm{E} 1 * 64)$, retinopathy $(\mathrm{E} 1 * 3 *)$, neuropathy $(\mathrm{E} 1 * 4 *)$, peripheral angiopathy $(\mathrm{E} 1 * 5 *)$ and foot ulcer $(\mathrm{E} 1 * 73)$ ], co-morbidities (CVD, cancer, hypertensive disease and hyperlipidaemia disease), glycaemic control rate i.e. HbA1c (\%), eGFR $\left(\mathrm{mL} / \mathrm{min} / 1.73 \mathrm{~m}^{2}\right)$ and DM medication history (number of oral DM medications, and use of metformin, sulfonylureas, acarbose and insulin). The differences in baseline characteristics were compared using Student t-test for continuous variables and Pearson's chi-squared test for categorical variables. Standardised differences were also used to compare baseline characteristics between the treatment cohorts.

\section{Definition of outcomes and statistical analyses}

The efficacy and safety of SGLT2 inhibitors and DPP4 inhibitors were assessed as classes of drugs since the individual drugs within the drug classes have the same mechanism of action with comparable clinical effectiveness and safety [17]. The outcomes measured were glycaemic control during 91-365 days after initiation as patients were typically followed up every three months, and any-cause, cause-specific hospitalisations, and all-cause death during 31-365 days after initiation. The HbA1c result nearest to the treatment initiation date was used as the baseline while the result closest to the date of 365 days after initiation was used as the post-treatment data [12]. Patients with missing $\mathrm{HbA1c}$ results during the follow-up period were excluded from the analysis. Cause-specific hospitalisations with these admission diagnoses were included in the analyses: diabetic ketoacidosis (DKA) (ICD-10 AM: E1*1* e.g. E1111 T2DM with ketoacidosis, without coma), primary T2DM (E11E14), primary T2DM with kidney complications (E1*2*), incipient diabetic nephropathy $(\mathrm{E} 1 * 21)$, hypoglycaemia (E1*64), CVD (I00-I99) and heart failure (HF) (I50*) as a secondary outcome with previous HF hospitalisation included as a co-variate, and urinary tract infection (UTI) (N10, N12, N136, N151, N159, N30, N300, N308, N309, N390). Only the first hospitalisation of each outcome was included in the analysis. Subgroup analyses by ethnicity (Chinese, Malay and Indian) were also performed to assess potential differential effect of SGLT2 inhibitors on patient outcomes.

Each patient in the SGLT2 inhibitor-treated cohort was matched with a patient from the DPP4 inhibitor-treated cohort with the nearest propensity score (PS), to account for differences in baseline characteristics and enable a more homogeneous comparison. Patients were matched 1:1 on PS which was derived from a logistic model using all covariates described. This was similarly done in the subgroup analysis where PS was derived and matched within each ethnic group. The balance in the two cohorts was assessed using standardised differences (a value less than 0.1 indicates negligible differences) [22, 23]. Finally, modified Poisson regression models [24] were also used to estimate risk ratios (RRs) and 95\% confidence intervals (CIs) for the matched SGLT2 inhibitor and DPP4 inhibitor-treated cohorts with and without adjustment. P-values lower than 0.05 were considered to be statistically significant. All analyses were performed using Stata version 16.

To derive the healthcare costs saved due to improvements in patient outcomes associated with SGLT2 inhibitor use, a Markov model was used to estimate the cumulative number of deaths and hospitalisations avoided and quantify the costs saved over 10 years. Cost savings were quantified by multiplying the difference in hospitalisation rates between the treatment cohorts by the number of patients on SGLT2 inhibitors and mean T2DM hospitalisation cost (assumed to remain unchanged). This difference in hospitalisation rate was applied across the years, with prevalent cases rolled over from the preceding year plus the incident cases in the current year. In addition, adjustments were made on the projected patient numbers excluding those due to deaths. These analyses were performed using Microsoft Excel.

\section{Results}

\section{Baseline demographics and clinical characteristics}

There were 71,587 eligible patients with outcomes measured 31-365 days after initiation. After excluding those below 30 years, non-residents, with missing information on gender or age, and those with a death record within 30 days of treatment initiation, 67,556 patients remained. Most patients were initiators of DPP4 inhibitors (about 77\%). Before matching, the two cohorts differed significantly on most baseline characteristics, with absolute standardised difference greater than 0.1. Patients in the SGLT2 inhibitor-treated cohort were younger compared to the DPP4 inhibitor-treated cohort (mean age 56 years vs. 63 years). There were more patients in the SGLT2 inhibitor-treated cohort with body weight $80 \mathrm{~kg}$ and above (21\% vs. $15 \%)$. In addition, more patients on SGLT2 inhibitors had disease duration of less than 5 years (31\% vs. $21 \%$ ) and fewer DM complications prior to treatment initiation (e.g. $2 \%$ vs. $14 \%$ for DM-kidney complications). However, more patients on SGLT2 inhibitors were using multiple oral drugs ( $39 \%$ vs. $29 \%$ on two drugs), metformin (64\% vs. $44 \%$ ) and insulin (21\% vs. $19 \%$ ) than DPP4 inhibitor-treated cohort. After PS matching, 15,207 comparable patients remained in each cohort with outcomes measured 31-365 days after initiation (Table 1 and Fig. 1). The results on the 35,694 eligible patients with outcomes measured 91-365 days after initiation and 5495 
comparable patients in each cohort after matching are provided in Appendix Table 5 and Fig. 2. The baseline characteristics of patients from different ethnic groups are also reported in Appendix Tables 6, 7, 8, 9, 10 and 11. The characteristics were well balanced after matching between the two cohorts.

\section{Glycaemic control}

In the matched cohort, SGLT2 inhibitor initiation was associated with a significantly lower mean $\mathrm{HbA} 1 \mathrm{c}$ than those initiated on DPP4 inhibitors (7.54\% vs. $7.68 \%, p<0.001)$. A higher proportion of patients also achieved good glycaemic control, HbA1c below 7\% (40.8\% SGLT2 vs. $37.5 \%$ DPP4) with a RR of 1.09 (95\% CI 1.04, 1.14). They were also less likely to report poor glycaemic control with HbA1c above $8 \%$ (RR 0.88; 95\% CI 0.83, 0.94). The difference between treatment groups was however not statistically significant in patients with $\mathrm{HbA} 1 \mathrm{c}$ between $7 \%$ and $8 \%$ in the overall cohort (Table 2). Similar results were observed only in patients of Chinese ethnicity while no significant difference were observed in patients of Malay and Indian ethnicity except lower risk of having $\mathrm{HbA} 1 \mathrm{c}$ between $7 \%$ and $8 \%$ in Indian patients on SGLT2 inhibitors.

\section{Safety outcomes}

In terms of safety outcomes, patients initiating SGLT2 inhibitors were not at higher risk of experiencing DKA compared to DPP4 inhibitors (Table 3). This was similarly observed for risk of DKA hospitalisation with length of stay seven days and longer. The risks of hospitalisation for hypoglycaemia (RR 0.69; 95\% CI 0.59, 0.82) were reduced with SGLT2 inhibitors and there was no increased risk of UTI hospitalisations (RR 0.52; 95\% CI 0.43, 0.63). These results were also observed across all ethnic groups except in patients of Malay ethnicity. The risk of DKA was significantly reduced in this ethnic group while no significant difference was observed in the occurrence of hypoglycaemia hospitalisations with SGLT2 inhibitor initiation.

\section{Hospitalisations and deaths}

In addition, SGLT2 inhibitors were associated with fewer hospitalisations and deaths up to one-year post-initiation compared to DPP4 inhibitors (Table 4). Any-cause and cause-specific hospitalisations ranged between 12\% (any hospitalisation) and 72\% (hospitalised for DM-related kidney complications) lower in the SGLT2 inhibitor-treated cohort compared to the DPP4 inhibitor-treated cohort. However, there was no difference in risk of diabetic nephropathy (except in patients of Indian ethnicity) and CVD hospitalisation between the treatment cohorts. Lower risk of all-cause mortality was observed among patients initiating SGLT2 inhibitors versus DPP4 inhibitors, with RR of 0.66 (95\% CI $0.51,0.85$ ). Circulatory system diseases, neoplasms and respiratory diseases were the most common causes of death. The lower risk of hospitalisations and deaths associated with SGLT2 inhibitors were similarly observed in patients of Chinese and Indian ethnicity (except risk of all-cause death was not statistically significant). In patients of Malay ethnicity, only hospitalisation risk for DM-related kidney complications was significantly reduced in patients on SGLT2 inhibitors compared to those on DPP4 inhibitors.

In the secondary analysis on hospitalisations for HF, patients on SGLT2 inhibitors were less likely to be hospitalised compared to DPP4 inhibitor initiators (RR 0.78; 95\% CI 0.63, 0.95) (Table 4). Among patients of Chinese ethnicity, a lower risk of HF hospitalisation was also observed in those initiating SGLT2 inhibitors compared to DPP4 inhibitors. There were no significant differences observed in patients of Malay or Indian ethnicity.

\section{Healthcare savings}

These benefits associated with SGLT2 inhibitors versus DPP4 inhibitors would lead to about 1261 deaths avoided and 8691 fewer hospitalisations. This contributes to more than $\$ 50$ million saved over 10 years.

\section{Discussion}

This is the first national real-world study in Singapore that evaluated the potential impact of ethnicity on the effects of SGLT2 inhibitors and DPP4 inhibitors. PS matching was performed to balance baseline characteristics between the treatment groups to minimise bias. In addition, improvements in patient outcomes associated with SGLT2 inhibitor initiation was also translated to healthcare cost savings to the system.

Our findings are consistent with other real-world studies and clinical trials showing SGLT2 inhibitor initiation to be associated with a higher likelihood of achieving $\mathrm{HbA} 1 \mathrm{c}$ targets compared to DPP4 inhibitor initiation (40.8\% vs. $37.5 \%)$. Locally, a single-centre retrospective cohort study of 57 patients also reported that patients on canagliflozin were more likely to attain $\mathrm{HbA} 1 \mathrm{c}$ levels below 7\% than patients in the sitagliptin group $(13.6 \%$ vs. $8.6 \%)$ at 24 -week follow-up [18]. Another prospective Canadian registry study assessing outcomes associated with canagliflozin observed that more patients achieved HbA1c below 7\% over time, reaching $38.8 \%$ by 12 months [25] which is similar to our findings of $40.8 \%$ up to one year follow-up. Similar findings were reported in real-world studies conducted in the US [26, 27]. In addition to canagliflozin, dapagliflozin also 
Table 1 Comparison of baseline characteristics in two treatment cohorts before and after PS matching

\begin{tabular}{|c|c|c|c|c|c|c|}
\hline \multirow[t]{2}{*}{ Variables } & \multicolumn{3}{|c|}{ Unmatched cohorts } & \multicolumn{3}{|l|}{ Matched cohorts } \\
\hline & $\begin{array}{l}\text { DPP4 inhibitors } \\
(\mathrm{n}=52,349)\end{array}$ & $\begin{array}{l}\text { SGLT2 inhibitors } \\
(\mathrm{n}=15,207)\end{array}$ & d & $\begin{array}{l}\text { DPP4 inhibitors } \\
(\mathrm{n}=15,207)\end{array}$ & $\begin{array}{l}\text { SGLT2 inhibitors } \\
(\mathrm{n}=15,207)\end{array}$ & d \\
\hline Age (years), mean $\pm S D$ & $\begin{array}{l}62.9 \pm 11.6 \\
\mathrm{n}(\%)\end{array}$ & $\begin{array}{l}56.3 \pm 10.2 \\
\mathrm{n}(\%)\end{array}$ & 0.110 & $\begin{array}{l}57.3 \pm 10.8 \\
\mathrm{n}(\%)\end{array}$ & $\begin{array}{l}56.3 \pm 10.2 \\
\mathrm{n}(\%)\end{array}$ & 0.016 \\
\hline \multicolumn{7}{|l|}{ Setting of initiation } \\
\hline Hospitals & $22,669(43.3 \%)$ & $5535(36.4 \%)$ & 0.140 & $5519(36.3 \%)$ & $5535(36.4 \%)$ & 0.002 \\
\hline Primary care clinics & $29,680(56.7 \%)$ & $9672(63.6 \%)$ & 0.141 & $9688(63.7 \%)$ & $9672(63.6 \%)$ & 0.002 \\
\hline \multicolumn{7}{|l|}{ Year of initiation } \\
\hline 2015 & $6933(13.2 \%)$ & $406(2.7 \%)$ & 0.400 & $347(2.3 \%)$ & $406(2.7 \%)$ & 0.055 \\
\hline 2016 & $12,425(23.7 \%)$ & $734(4.8 \%)$ & 0.560 & $721(4.7 \%)$ & $734(4.8 \%)$ & 0.004 \\
\hline 2017 & $16,240(31.0 \%)$ & $5515(36.3 \%)$ & 0.111 & $5960(39.2 \%)$ & $5515(36.3 \%)$ & 0.060 \\
\hline 2018 & $16,751(32.0 \%)$ & $8552(56.2 \%)$ & 0.503 & $8179(53.8 \%)$ & $8552(56.2 \%)$ & 0.049 \\
\hline Gender (male) & $27,175(51.9 \%)$ & $8361(55.0 \%)$ & 0.062 & $8265(54.4 \%)$ & $8361(55.0 \%)$ & 0.013 \\
\hline \multicolumn{7}{|l|}{ Ethnicity } \\
\hline Chinese & $33,071(63.2 \%)$ & $9199(60.5 \%)$ & 0.060 & $9204(60.5 \%)$ & $9199(60.5 \%)$ & 0.001 \\
\hline Indian & $7326(14.0 \%)$ & $2461(16.2 \%)$ & 0.061 & $2450(16.1 \%)$ & $2461(16.2 \%)$ & 0.002 \\
\hline Malay & 7297 (13.9\%) & $2087(13.7 \%)$ & 0.010 & $2124(14.0 \%)$ & $2087(13.7 \%)$ & 0.007 \\
\hline Others & $4655(8.9 \%)$ & $1460(9.6 \%)$ & 0.025 & $1429(9.4 \%)$ & $1460(9.6 \%)$ & 0.007 \\
\hline \multicolumn{7}{|l|}{ Residence } \\
\hline $\mathrm{SC}$ & $50,326(96.1 \%)$ & $14,423(94.8 \%)$ & 0.060 & $14,445(95.0 \%)$ & $14,423(94.8 \%)$ & 0.007 \\
\hline PR & $2023(3.9 \%)$ & $784(5.2 \%)$ & 0.063 & $762(5.0 \%)$ & $784(5.2 \%)$ & 0.007 \\
\hline \multicolumn{7}{|l|}{ SES category } \\
\hline Maximum subsidy & $23,281(44.5 \%)$ & $4751(31.2 \%)$ & 0.280 & $4842(31.8 \%)$ & $4751(31.2 \%)$ & 0.013 \\
\hline Some subsidy & $1059(2.0 \%)$ & $436(2.9 \%)$ & 0.055 & $403(2.7 \%)$ & $436(2.9 \%)$ & 0.013 \\
\hline Minimum subsidy & $1338(2.6 \%)$ & $500(3.3 \%)$ & 0.043 & $506(3.3 \%)$ & $500(3.3 \%)$ & 0.002 \\
\hline NA & $26,671(51.0 \%)$ & $9520(62.6 \%)$ & 0.237 & $9456(62.2 \%)$ & $9520(62.6 \%)$ & 0.009 \\
\hline \multicolumn{7}{|l|}{ Weight (kilograms) } \\
\hline$<65$ & $15,673(29.9 \%)$ & $3334(21.9 \%)$ & 0.180 & $3424(22.5 \%)$ & $3334(21.9 \%)$ & 0.014 \\
\hline $65-79$ & $13,110(25.0 \%)$ & $3729(24.5 \%)$ & 0.010 & $3841(25.3 \%)$ & $3729(24.5 \%)$ & 0.017 \\
\hline$\geq 80$ & $7692(14.7 \%)$ & $3178(20.9 \%)$ & 0.163 & $2942(19.4 \%)$ & $3178(20.9 \%)$ & 0.039 \\
\hline NA & $15,874(30.3 \%)$ & $4966(32.7 \%)$ & 0.050 & $5000(32.9 \%)$ & $4966(32.7 \%)$ & 0.005 \\
\hline \multicolumn{7}{|c|}{ Cigarette smoking (number of cigarettes per day) } \\
\hline Non-smoker & $19,379(37.0 \%)$ & $5046(33.2 \%)$ & 0.080 & $5092(33.5 \%)$ & $5046(33.2 \%)$ & 0.006 \\
\hline $1-9$ & $1585(3.0 \%)$ & $522(3.4 \%)$ & 0.023 & $506(3.3 \%)$ & $522(3.4 \%)$ & 0.006 \\
\hline$\geq 10$ & $3092(5.9 \%)$ & $1003(6.6 \%)$ & 0.028 & $983(6.5 \%)$ & $1003(6.6 \%)$ & 0.006 \\
\hline NA & $28,293(54.1 \%)$ & $8636(56.8 \%)$ & 0.055 & $8626(56.7 \%)$ & $8636(56.8 \%)$ & 0.001 \\
\hline \multicolumn{7}{|l|}{ Diastolic BP (mmHg) } \\
\hline$<65$ & $11,053(21.1 \%)$ & $2638(17.4 \%)$ & 0.100 & $2683(17.6 \%)$ & $2638(17.4 \%)$ & 0.008 \\
\hline $65-89$ & $27,929(53.4 \%)$ & $8101(53.3 \%)$ & 0.002 & $8034(52.8 \%)$ & $8101(53.3 \%)$ & 0.009 \\
\hline$\geq 90$ & $1882(3.6 \%)$ & $582(3.8 \%)$ & 0.012 & $599(3.9 \%)$ & $582(3.8 \%)$ & 0.006 \\
\hline NA & $11,485(21.9 \%)$ & $3886(25.6 \%)$ & 0.085 & $3891(25.6 \%)$ & $3886(25.6 \%)$ & 0.001 \\
\hline \multicolumn{7}{|l|}{ Systolic BP (mmHg) } \\
\hline$<130$ & $16,336(31.2 \%)$ & $4644(30.5 \%)$ & 0.020 & $4575(30.1 \%)$ & $4644(30.5 \%)$ & 0.010 \\
\hline $130-139$ & $11,575(22.1 \%)$ & $3393(22.3 \%)$ & 0.005 & $3432(22.6 \%)$ & $3393(22.3 \%)$ & 0.006 \\
\hline$\geq 140$ & $12,953(24.7 \%)$ & $3284(21.6 \%)$ & 0.070 & $3309(21.8 \%)$ & $3284(21.6 \%)$ & 0.004 \\
\hline NA & $11,485(21.9 \%)$ & $3886(25.6 \%)$ & 0.085 & $3891(25.6 \%)$ & $3886(25.6 \%)$ & 0.001 \\
\hline \multicolumn{7}{|c|}{ Duration with diabetes (years) } \\
\hline $0-4$ & $10,897(20.8 \%)$ & $4773(31.4 \%)$ & 0.242 & $4473(29.4 \%)$ & $4773(31.4 \%)$ & 0.043 \\
\hline
\end{tabular}


Table 1 (continued)

\begin{tabular}{|c|c|c|c|c|c|c|}
\hline \multirow[t]{2}{*}{ Variables } & \multicolumn{3}{|c|}{ Unmatched cohorts } & \multicolumn{3}{|l|}{ Matched cohorts } \\
\hline & $\begin{array}{l}\text { DPP4 inhibitors } \\
(\mathrm{n}=52,349)\end{array}$ & $\begin{array}{l}\text { SGLT2 inhibitors } \\
(\mathrm{n}=15,207)\end{array}$ & $\mathrm{d}$ & $\begin{array}{l}\text { DPP4 inhibitors } \\
(\mathrm{n}=15,207)\end{array}$ & $\begin{array}{l}\text { SGLT2 inhibitors } \\
(\mathrm{n}=15,207)\end{array}$ & $\mathrm{d}$ \\
\hline $5-9$ & $16,189(30.9 \%)$ & $4188(27.5 \%)$ & 0.080 & $4202(27.6 \%)$ & $4188(27.5 \%)$ & 0.002 \\
\hline$\geq 10$ & $24,482(46.8 \%)$ & $5837(38.4 \%)$ & 0.170 & $6151(40.5 \%)$ & $5837(38.4 \%)$ & 0.042 \\
\hline NA & $781(1.5 \%)$ & $409(2.7 \%)$ & 0.084 & $381(2.5 \%)$ & $409(2.7 \%)$ & 0.011 \\
\hline \multicolumn{7}{|c|}{ Diagnoses for hospitalisation 1-365 days prior to initiation } \\
\hline Any hospitalisation & $18,783(35.9 \%)$ & $3130(20.6 \%)$ & 0.350 & $3207(21.1 \%)$ & $3130(20.6 \%)$ & 0.013 \\
\hline DM-kidney complications & $7051(13.5 \%)$ & $368(2.4 \%)$ & 0.420 & $375(2.5 \%)$ & $368(2.4 \%)$ & 0.003 \\
\hline Retinopathy & $2651(5.1 \%)$ & $513(3.4 \%)$ & 0.080 & $538(3.5 \%)$ & $513(3.4 \%)$ & 0.009 \\
\hline Neuropathy & $802(1.5 \%)$ & $75(0.5 \%)$ & 0.100 & $70(0.5 \%)$ & $75(0.5 \%)$ & 0.004 \\
\hline Peripheral angiopathy & $1285(2.5 \%)$ & $86(0.6 \%)$ & 0.160 & $79(0.5 \%)$ & $86(0.6 \%)$ & 0.007 \\
\hline Poor control & $6409(12.2 \%)$ & $856(5.6 \%)$ & 0.230 & $894(5.9 \%)$ & $856(5.6 \%)$ & 0.011 \\
\hline Hypoglycaemia & $3072(5.9 \%)$ & $173(1.1 \%)$ & 0.260 & $170(1.1 \%)$ & $173(1.1 \%)$ & 0.002 \\
\hline Insulin resistance & $15,477(29.6 \%)$ & $2236(14.7 \%)$ & 0.360 & $2270(14.9 \%)$ & $2236(14.7 \%)$ & 0.006 \\
\hline Foot ulcer & $1203(2.3 \%)$ & $124(0.8 \%)$ & 0.120 & $139(0.9 \%)$ & $124(0.8 \%)$ & 0.010 \\
\hline \multicolumn{7}{|l|}{$\mathrm{HbA1c}(\%)$} \\
\hline$<7$ & $4983(9.5 \%)$ & $1261(8.3 \%)$ & 0.040 & $1253(8.2 \%)$ & $1261(8.3 \%)$ & 0.002 \\
\hline $7-8.9$ & $19,638(37.5 \%)$ & $5264(34.6 \%)$ & 0.060 & $5328(35.0 \%)$ & $5264(34.6 \%)$ & 0.009 \\
\hline$\geq 9$ & $11,716(22.4 \%)$ & $3378(22.2 \%)$ & 0.004 & $3300(21.7 \%)$ & $3378(22.2 \%)$ & 0.012 \\
\hline NA & $16,012(30.6 \%)$ & $5304(34.9 \%)$ & 0.092 & $5326(35.0 \%)$ & $5304(34.9 \%)$ & 0.003 \\
\hline \multicolumn{7}{|l|}{$\mathrm{eGFR}\left(\mathrm{mL} / \mathrm{min} / 1.73 \mathrm{~m}^{2}\right)$} \\
\hline$<60$ & $7499(14.3 \%)$ & $588(3.9 \%)$ & 0.370 & $526(3.5 \%)$ & $588(3.9 \%)$ & 0.022 \\
\hline $60-89$ & $6973(13.3 \%)$ & $1964(12.9 \%)$ & 0.010 & $2041(13.4 \%)$ & $1964(12.9 \%)$ & 0.015 \\
\hline$\geq 90$ & $7202(13.8 \%)$ & $2622(17.2 \%)$ & 0.096 & $2709(17.8 \%)$ & $2622(17.2 \%)$ & 0.015 \\
\hline NA & $30,675(58.6 \%)$ & $10,033(66.0 \%)$ & 0.153 & $9931(65.3 \%)$ & $10,033(66.0 \%)$ & 0.014 \\
\hline \multicolumn{7}{|c|}{ Diagnoses in 3 years prior to initiation (co-morbid conditions) } \\
\hline Any CVD & $23,257(44.4 \%)$ & $4568(30.0 \%)$ & 0.300 & $4614(30.3 \%)$ & $4568(30.0 \%)$ & 0.007 \\
\hline Any cancer & $1676(3.2 \%)$ & $197(1.3 \%)$ & 0.130 & $207(1.4 \%)$ & $197(1.3 \%)$ & 0.005 \\
\hline Hypertensive disease & $21,286(40.7 \%)$ & $3859(25.4 \%)$ & 0.330 & $3935(25.9 \%)$ & $3859(25.4 \%)$ & 0.011 \\
\hline Hyperlipidaemia & $19,579(37.4 \%)$ & $3727(24.5 \%)$ & 0.280 & $3766(24.8 \%)$ & $3727(24.5 \%)$ & 0.006 \\
\hline \multicolumn{7}{|c|}{ Medication history of DM drugs $1-365$ days prior to initiation } \\
\hline \multicolumn{7}{|l|}{ Number of oral DM drugs } \\
\hline No records & $12,738(24.3 \%)$ & $3324(21.9 \%)$ & 0.060 & $3672(24.2 \%)$ & $3324(21.9 \%)$ & 0.054 \\
\hline 1 & $20,987(40.1 \%)$ & $5277(34.7 \%)$ & 0.110 & $5001(32.9 \%)$ & $5277(34.7 \%)$ & 0.038 \\
\hline 2 & $15,281(29.2 \%)$ & $5861(38.5 \%)$ & 0.199 & $5785(38.0 \%)$ & $5861(38.5 \%)$ & 0.010 \\
\hline$\geq 3$ & $3343(6.4 \%)$ & $745(4.9 \%)$ & 0.070 & $749(4.9 \%)$ & $745(4.9 \%)$ & 0.001 \\
\hline MET & $23,075(44.1 \%)$ & $9711(63.9 \%)$ & 0.405 & $9199(60.5 \%)$ & $9711(63.9 \%)$ & 0.070 \\
\hline SU & $33,520(64.0 \%)$ & $8497(55.9 \%)$ & 0.170 & $8612(56.6 \%)$ & $8497(55.9 \%)$ & 0.015 \\
\hline Acarbose & $3690(7.1 \%)$ & $661(4.4 \%)$ & 0.120 & $688(4.5 \%)$ & $661(4.4 \%)$ & 0.008 \\
\hline Insulin & $9971(19.1 \%)$ & $3112(20.5 \%)$ & 0.035 & $2966(19.5 \%)$ & $3112(20.5 \%)$ & 0.024 \\
\hline
\end{tabular}

DPP4: dipeptidyl peptidase 4; SGLT2: sodium-glucose co-transporter 2; d: standardised difference; SD: standard deviation; SC: Singapore citizen; PR: Singapore permanent resident; SES: socioeconomic status; NA: not applicable; BP: blood pressure; DM: diabetes mellitus; HbA1c: haemoglobin A1c; eGFR: estimated glomerular filtration rate; CVD: cardiovascular disease; MET: metformin; SU: sulfonylureas 
Fig. 1 Selection of study population for outcomes measured 31-365 days after initiation
Table 2 RR and associated 95\% CIs for glycaemic control in two treatment cohorts after treatment initiation

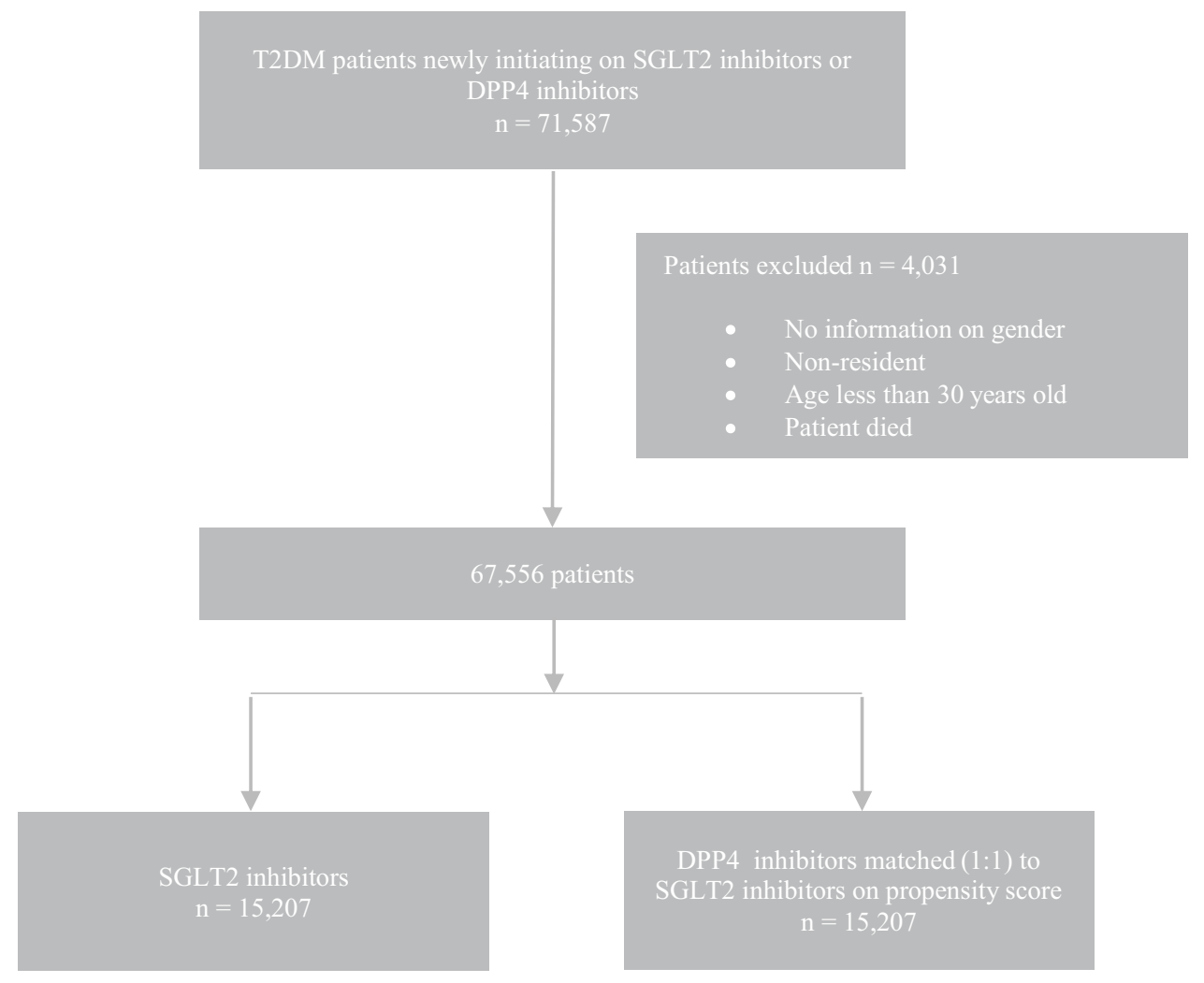

\begin{tabular}{|c|c|c|c|c|}
\hline Outcomes, n(\%) & DPP4 inhibitors & SGLT2 inhibitors & Unadjusted RR (95\% CI) & Adjusted RR $(95 \% \mathrm{CI})^{\wedge}$ \\
\hline \multicolumn{5}{|c|}{ All patients (5495 matched patients from each treatment cohort) } \\
\hline $\mathrm{HbA} 1 \mathrm{c}<7 \%$ & $2062(37.5 \%)$ & $2240(40.8 \%)$ & $1.09(1.04,1.14)$ & $1.09(1.04,1.14)$ \\
\hline $\mathrm{HbA} 1 \mathrm{c} 7-8 \%$ & $1740(31.7 \%)$ & $1761(32.1 \%)$ & $1.01(0.96,1.07)$ & $1.01(0.96,1.07)$ \\
\hline $\mathrm{HbA} 1 \mathrm{c}>8 \%$ & $1693(30.8 \%)$ & $1494(27.2 \%)$ & $0.88(0.83,0.94)$ & $0.88(0.83,0.94)$ \\
\hline \multicolumn{5}{|c|}{ Chinese (3365 matched patients from each treatment cohort) } \\
\hline $\mathrm{HbA} 1 \mathrm{c}<7 \%$ & $1264(37.6 \%)$ & $1382(41.1 \%)$ & $1.09(1.03,1.16)$ & $1.09(1.03,1.16)$ \\
\hline $\mathrm{HbA} 1 \mathrm{c} 7-8 \%$ & $1138(33.8 \%)$ & $1150(34.2 \%)$ & $1.01(0.95,1.08)$ & $1.01(0.95,1.08)$ \\
\hline $\mathrm{HbA} 1 \mathrm{c}>8 \%$ & $963(28.6 \%)$ & $833(24.8 \%)$ & $0.87(0.80,0.94)$ & $0.87(0.80,0.94)$ \\
\hline \multicolumn{5}{|c|}{ Indian (905 matched patients from each treatment cohort) } \\
\hline $\mathrm{HbA} 1 \mathrm{c}<7 \%$ & $318(35.1 \%)$ & $357(39.5 \%)$ & $1.12(1.00,1.27)$ & $1.11(0.99,1.25)$ \\
\hline $\mathrm{HbA} 1 \mathrm{c} 7-8 \%$ & $294(32.5 \%)$ & $254(28.1 \%)$ & $0.86(0.75,0.99)$ & $0.86(0.75,0.99)$ \\
\hline $\mathrm{HbA} 1 \mathrm{c}>8 \%$ & $293(32.4 \%)$ & $294(32.5 \%)$ & $1.00(0.88,1.15)$ & $1.00(0.88,1.14)$ \\
\hline \multicolumn{5}{|c|}{ Malay (745 matched patients from each treatment cohort) } \\
\hline $\mathrm{HbA} 1 \mathrm{c}<7 \%$ & $280(37.6 \%)$ & $303(40.7 \%)$ & $1.08(0.95,1.23)$ & $1.07(0.94,1.21)$ \\
\hline $\mathrm{HbA} 1 \mathrm{c} 7-8 \%$ & $222(29.8 \%)$ & $218(29.3 \%)$ & $0.98(0.84,1.15)$ & $0.98(0.84,1.15)$ \\
\hline $\mathrm{HbA} 1 \mathrm{c}>8 \%$ & $243(32.6 \%)$ & $224(30.1 \%)$ & $0.92(0.79,1.07)$ & $0.92(0.80,1.07)$ \\
\hline
\end{tabular}

$\wedge$ Adjusted for baseline $\mathrm{HbAlc}$ and year of initiation (for Indian and Malay patients)

HbA1c: haemoglobin A1c; DPP4: dipeptidyl peptidase 4; SGLT2: sodium-glucose co-transporter 2; RR: risk ratio; $\mathrm{CI}$ : confidence interval showed greater reductions in $\mathrm{HbA1c}$ than other oral antidiabetic drugs such as DPP4 inhibitors, with more patients attaining target glycaemic control or reduction in the realworld setting [28-30]. SGLT2 inhibitors also showed better glycaemic control than DPP4 inhibitors in clinical trials [31, 32]. A meta-analysis comprising 25 randomised controlled trials (RCTs) observed no statistically significant difference 
Table 3 RR and associated 95\% CIs for DKA, hypoglycaemia and UTI in two treatment cohorts after treatment initiation

\begin{tabular}{|c|c|c|c|}
\hline Outcomes, n (\%) & DPP4 inhibitors & SGLT2 inhibitors & $\mathrm{RR}(95 \% \mathrm{CI})$ \\
\hline \multicolumn{4}{|c|}{ All patients $(15,207$ matched patients from each treatment cohort) } \\
\hline DKA & $108(0.7 \%)$ & $83(0.6 \%)$ & $0.77(0.58,1.02)$ \\
\hline DKA hospitalisation with length of stay $\geq 7$ days & $47(0.3 \%)$ & $44(0.3 \%)$ & $0.94(0.62,1.41)$ \\
\hline Hospitalised for hypoglycaemia & $347(2.3 \%)$ & $241(1.6 \%)$ & $0.69(0.59,0.82)$ \\
\hline Hospitalised for UTI & $332(2.2 \%)$ & $173(1.1 \%)$ & $0.52(0.43,0.63)$ \\
\hline \multicolumn{4}{|c|}{ Chinese (9199 matched patients from each treatment cohort) } \\
\hline DKA & $55(0.6 \%)$ & $51(0.6 \%)$ & $0.93(0.63,1.36)$ \\
\hline DKA hospitalisation with length of stay $\geq 7$ days & $27(0.3 \%)$ & $25(0.3 \%)$ & $0.93(0.54,1.59)$ \\
\hline Hospitalised for hypoglycaemia & $189(2.1 \%)$ & $124(1.4 \%)$ & $0.66(0.52,0.82)$ \\
\hline Hospitalised for UTI & $166(1.8 \%)$ & $95(1.0 \%)$ & $0.57(0.45,0.74)$ \\
\hline \multicolumn{4}{|c|}{ Indian (2461 matched patients from each treatment cohort) } \\
\hline DKA & $22(0.9 \%)$ & $11(0.5 \%)$ & $0.50(0.24,1.03)$ \\
\hline DKA hospitalisation with length of stay $\geq 7$ days & $10(0.4 \%)$ & $6(0.2 \%)$ & $0.60(0.22,1.65)$ \\
\hline Hospitalised for hypoglycaemia & $94(3.8 \%)$ & $48(2.0 \%)$ & $0.51(0.36,0.72)$ \\
\hline Hospitalised for UTI & $74(3.0 \%)$ & $39(1.6 \%)$ & $0.53(0.36,0.77)$ \\
\hline \multicolumn{4}{|c|}{ Malay (2087 matched patients from each treatment cohort) } \\
\hline DKA & $30(1.4 \%)$ & $15(0.7 \%)$ & $0.50(0.27,0.93)$ \\
\hline DKA hospitalisation with length of stay $\geq 7$ days & $12(0.6 \%)$ & $10(0.5 \%)$ & $0.83(0.36,1.92)$ \\
\hline Hospitalised for hypoglycaemia & $49(2.4 \%)$ & $44(2.1 \%)$ & $0.90(0.60,1.34)$ \\
\hline Hospitalised for UTI & $59(2.8 \%)$ & $24(1.2 \%)$ & $0.41(0.25,0.65)$ \\
\hline
\end{tabular}

DPP4: dipeptidyl peptidase 4; SGLT2: sodium-glucose co-transporter 2; RR: risk ratio; CI: confidence interval; DKA: diabetic ketoacidosis; UTI: urinary tract infection between the treatment groups but there was substantial heterogeneity across studies $\left(\mathrm{I}^{2}=62 \%\right)[33]$.

As expected, the relative efficacy of treatments differed across ethnic groups. Although SGLT2 inhibitor use increased the likelihood of achieving target glycaemic control in patients of Chinese ethnicity, this was not observed in patients of Malay and Indian ethnicity. This is consistent with the literature that diabetes control is more optimal among the Chinese compared to Malays and Indians [34], thus highlighting the need to consider ethnicity in diabetes management and when assessing clinical outcomes. It is also important to realise that ethnicity is affected by genetic and environmental factors such as body fat distribution, adipose tissue function, differences in insulin secretion levels and insulin sensitivity, health beliefs and dietary habits [34, 35], forming a complex interplay of risk factors.

In terms of safety outcomes, the literature was mixed, with some studies reporting increased DKA risk with SGLT2 inhibitors and other studies reporting no increase. Our study did not observe a higher risk of hospitalisation for DKA with SGLT2 inhibitors. Similarly, another nationwide retrospective cohort study in Korea did not observe an increase in DKA risk in the SGLT2 inhibitor-treated group [hazard ratio (HR) 0.956; 95\% CI 0.581, 1.572; $\mathrm{p}=0.996$ ] after PS matching [13]. The risk of DKA was also not higher in the SGLT2 inhibitor-treated group in a meta-analysis consisting of 81 trials, with Mantel-Haenszel odds ratio
(OR) of $1.14(95 \%$ CI $0.45,2.88 ; \mathrm{p}=0.78)$ [36]. Two other meta-analyses [37, 38], the Empagliflozin Cardiovascular Outcome Event Trial in Type 2 Diabetes Mellitus Patients - Removing Excess Glucose (EMPA-REG OUTCOME) trial [39] and Canagliflozin Cardiovascular Assessment Study (CANVAS) programme [40] also reported similar results. On the other hand, a retrospective observational study in the US (HR 2.2; 95\% CI 1.4, 3.6) and a cohort study on Scandinavian countries (HR 2.14; 95\% CI 1.17, 4.09) found treatment with SGLT2 inhibitors to be associated with higher DKA risk than DPP4 inhibitors with PS matching [41, 42]. Clinicians may need to continue monitoring patients who are initially starting SGLT2 inhibitors, in particular, euglycaemic DKA which can be easily missed due to normal glucose levels [43-45] or when there are symptoms such as nausea and vomiting which may indicate ketoacidosis [46].

Hypoglycaemia results in our study are also consistent with those in the published literature. The risk of hospitalisations for hypoglycaemia was $31 \%$ lower in patients initiating SGLT2 inhibitors compared to DPP4 inhibitors in our study. This effect was similarly observed in the ethnic subgroups but was not statistically significant in patients of Malay ethnicity. A meta-analysis of nine RCTs also reported lower risk of hypoglycaemia with SGLT2 inhibitors (OR $0.48 ; 95 \%$ CI $0.28,0.82 ; p=0.008$ ) [38]. This was also observed in realworld studies with patients receiving dapagliflozin reporting lower rates of hypoglycaemia than other oral drugs $(0.6 \%$ 
Table 4 RR and associated 95\% CIs for hospitalisations and deaths in two treatment cohorts after treatment initiation

\begin{tabular}{|c|c|c|c|}
\hline Outcomes, $\mathrm{n}(\%)$ & DPP4 inhibitors & SGLT2 inhibitors & $\mathrm{RR}(95 \% \mathrm{CI})$ \\
\hline \multicolumn{4}{|c|}{ All patients $(15,207$ matched patients from each treatment cohort) } \\
\hline Any hospitalisation & $2830(18.6 \%)$ & $2489(16.4 \%)$ & $0.88(0.84,0.92)$ \\
\hline Hospitalised for DM (principal diagnosis) & $546(3.6 \%)$ & $336(2.2 \%)$ & $0.62(0.54,0.70)$ \\
\hline Hospitalised for DM-related kidney complications & $156(1.0 \%)$ & $44(0.3 \%)$ & $0.28(0.20,0.39)$ \\
\hline Hospitalised for diabetic nephropathy & $34(0.2 \%)$ & $37(0.2 \%)$ & $1.09(0.68,1.73)$ \\
\hline Hospitalised for CVD & $534(3.5 \%)$ & $570(3.8 \%)$ & $1.07(0.95,1.20)$ \\
\hline Hospitalised for $\mathrm{HF}$ & $211(1.4 \%)$ & $164(1.1 \%)$ & $0.78(0.63,0.95)$ \\
\hline All-cause death & $151(1.0 \%)$ & $100(0.7 \%)$ & $0.66(0.51,0.85)$ \\
\hline \multicolumn{4}{|c|}{ Chinese (9199 matched patients from each treatment cohort) } \\
\hline Any hospitalisation & $1467(16.0 \%)$ & $1263(13.7 \%)$ & $0.86(0.80,0.92)$ \\
\hline Hospitalised for DM (principal diagnosis) & $280(3.0 \%)$ & $145(1.6 \%)$ & $0.52(0.42,0.63)$ \\
\hline Hospitalised for DM-related kidney complications & $92(1.0 \%)$ & $20(0.2 \%)$ & $0.22(0.13,0.35)$ \\
\hline Hospitalised for diabetic nephropathy & $10(0.1 \%)$ & $18(0.2 \%)$ & $1.80(0.83,3.90)$ \\
\hline Hospitalised for CVD & $299(3.3 \%)$ & $293(3.2 \%)$ & $0.98(0.84,1.15)$ \\
\hline Hospitalised for HF & $124(1.4 \%)$ & $76(0.8 \%)$ & $0.61(0.46,0.81)$ \\
\hline All-cause death & $87(1.0 \%)$ & $56(0.6 \%)$ & $0.64(0.46,0.90)$ \\
\hline \multicolumn{4}{|c|}{ Indian (2461 matched patients from each treatment cohort) } \\
\hline Any hospitalisation & $594(24.1 \%)$ & $521(21.2 \%)$ & $0.88(0.79,0.97)$ \\
\hline Hospitalised for DM (principal diagnosis) & $114(4.6 \%)$ & $65(2.6 \%)$ & $0.57(0.42,0.77)$ \\
\hline Hospitalised for DM-related kidney complications & $27(1.1 \%)$ & $9(0.4 \%)$ & $0.33(0.16,0.71)$ \\
\hline Hospitalised for diabetic nephropathy & $15(0.6 \%)$ & $5(0.2 \%)$ & $0.33(0.12,0.92)$ \\
\hline Hospitalised for CVD & $115(4.7 \%)$ & $126(5.1 \%)$ & $1.10(0.86,1.40)$ \\
\hline Hospitalised for $\mathrm{HF}$ & $47(1.9 \%)$ & $32(1.3 \%)$ & $0.68(0.44,1.06)$ \\
\hline All-cause death & $25(1.0 \%)$ & $17(0.7 \%)$ & $0.68(0.37,1.26)$ \\
\hline \multicolumn{4}{|c|}{ Malay (2087 matched patients from each treatment cohort) } \\
\hline Any hospitalisation & $476(22.8 \%)$ & $441(21.1 \%)$ & $0.93(0.83,1.04)$ \\
\hline Hospitalised for DM (principal diagnosis) & $101(4.8 \%)$ & $84(4.0 \%)$ & $0.83(0.63,1.10)$ \\
\hline Hospitalised for DM-related kidney complications & $26(1.3 \%)$ & $12(0.6 \%)$ & $0.46(0.23,0.91)$ \\
\hline Hospitalised for diabetic nephropathy & $5(0.2 \%)$ & $12(0.6 \%)$ & $2.40(0.85,6.80)$ \\
\hline Hospitalised for CVD & $82(3.9 \%)$ & $95(4.6 \%)$ & $1.16(0.87,1.55)$ \\
\hline Hospitalised for $\mathrm{HF}$ & $27(1.3 \%)$ & $40(1.9 \%)$ & $1.48(0.91,2.40)$ \\
\hline All-cause death & $32(1.5 \%)$ & $19(0.9 \%)$ & $0.59(0.34,1.04)$ \\
\hline
\end{tabular}

DPP4: dipeptidyl peptidase 4; SGLT2: sodium-glucose co-transporter 2; RR: risk ratio; CI: confidence interval; DM: diabetes mellitus; CVD: cardiovascular disease; HF: heart failure vs. 1.3\%) [28] and decreased risk of hypoglycaemia with SGLT2 inhibitors (HR 0.76; 95\% CI 0.65, 0.90; $\mathrm{p}=0.001$ ) [47]. A systematic review comprising 25 RCTs (RR, 0.99; 95\% CI 0.78, 1.26, $\mathrm{p}=0.92$ ) [33] and an additional RCT (4.0\% vs. 3.4\%) [31] found the risk or incidence of hypoglycaemic events to be similar between users of SGLT2 inhibitors and DPP4 inhibitors.

In addition, we observed that SGLT2 inhibitors did not increase the risk of UTI hospitalisations compared to DPP4 inhibitors in the overall cohort and across all ethnic groups. This is consistent with a large US cohort study of 123,752 matched patients on SGLT2 inhibitors and DPP4 inhibitors which also found a lower risk of UTI hospitalisations (HR $0.68 ; 95 \%$ CI $0.54,0.87$ ) [48]. Two meta-analyses did not report an increased risk of severe or non-severe UTI events with SGLT2 inhibitors [49, 50]. Another observational study in Australia similarly did not find a higher risk of UTI infections in SGLT2 inhibitor initiators (HR 0.90; 95\% CI 0.66, 1.24) [51]. Other studies also reported similar UTI rates between treatment groups $[31,37,52,53]$ while a pooled analysis (OR 1.15; 95\% CI 1.00, 1.33; p=0.047) [38] and a retrospective cohort study in Korea (HR 1.05; 95\% CI 1.00, $1.11 ; p=0.047$ ) [54] reported increased risk of UTIs with SGLT2 inhibitors which was borderline significant.

Our study also found that SGLT2 inhibitors reduced the risk of hospitalisations (except for CVD hospitalisations and hospitalisations for diabetic nephropathy) and all-cause death compared to DPP4 inhibitors. Other real-world studies also showed SGLT2 inhibitors were associated with a lower risk of all-cause death compared 
with other diabetes drugs (HR 0.51; 95\% CI 0.37, 0.70; $\mathrm{p}<0.001$ ) [55]. Furthermore, this finding was consistent across countries, ranging from $25 \%$ in Singapore to $68 \%$ reduced risk in Australia. The lower risk of death was attenuated when restricted to first new-user and using intention to treat approach (HR $0.65 ; 95 \%$ CI $0.60,0.71 ; \mathrm{p}<0.001$ ) [55], similar to our study findings of $34 \%$ reduced risk of death in the SGLT2 inhibitortreated cohort. Other observational studies [42, 56-58], clinical trials such as EMPA-REG OUTCOME trial [39] and CANVAS programme [40], and a meta-analysis [38] also reported a lower risk of all-cause death with SGLT2 inhibitors. A real-world study in Israel also reported reduced risk of hospitalisations (OR $0.662 ; 95 \%$ CI 0.564 , 0.776; $p<0.001)$ in patients initiating SGLT2 inhibitors compared with DPP4 inhibitors up to 24 weeks and its effects were similarly observed in the matched populations (OR 0.731; 95\% CI 0.603, 0.885; p = 0.001) [58]. As expected, the magnitude of decreased hospitalisation risk varied across ethnic groups with patients of Chinese ethnicity reporting greater reductions in hospitalisation and death risk than other ethnic groups in our study. This again highlights the importance of including ethnicity when assessing the impact of treatments on patient outcomes.

Although no significant differences were observed for CVD hospitalisations, SGLT2 inhibitor-treated patients were $22 \%$ less likely to be hospitalised for HF than DPP4 inhibitor-treated patients in our study. This is similarly observed in other retrospective observational studies in Korea (HR 0.66; 95\% CI 0.58, 0.75; p < 0.001) [59] and US (HR 0.68; 95\% CI $0.54,0.86 ; p=0.001$ ) [60]. A network meta-analysis study of 58 trials also reported reduced HF events with SGLT2 inhibitors (HR 0.55; 95\% CI 0.46, 0.67; $\mathrm{I}^{2}=19 \%$ ) [61]. Our findings are also consistent with those from the observational Comparative Effectiveness of Cardiovascular Outcomes in New Users of Sodium-Glucose Cotransporter-2 Inhibitors (CVD-REAL) 2 study comprising patients from six countries including Singapore. SGLT2 inhibitors were associated with $26 \%$ lower risk of HF hospitalisation than other oral and injectable glucose-lowering drugs (HR 0.74; 95\% CI $0.69,0.80$ ) [55]. However, statistically significant reduction was not observed in patients from Singapore (HR 0.58; $95 \%$ CI $0.34,1.00)$ likely due to the small sample size $(n=2222)$ [55]. The CVD-REAL Nordic study also observed a reduced risk of hospitalisations for HF with SGLT2 inhibitors compared to other diabetes drugs (HR 0.70; 95\% CI 0.61, 0.81; $\mathrm{p}<0.0001$ ) [47]. In a later CVD-REAL Nordic study comparing dapagliflozin and DPP4 inhibitors, similar findings on hospitalisation for HF were reported (HR 0.69; 95\% CI 0.57, 0.84; $\mathrm{p}<0.001$ ) [56]. A Scandinavian register based cohort study also found significant differences in HF events favouring SGLT2 inhibitors over DPP4 inhibitors (HR 0.66; 95\% CI 0.53, 0.81) [42].

Our study also estimated that the reduced risk of hospitalisations associated with SGLT2 inhibitors would translate to cumulative savings of more than $\$ 50$ million and 1261 deaths avoided over 10 years. Although the use of newer drugs such as SGLT2 inhibitors to improve glycaemic control would increase spending, these costs were offset by savings in the longer term from lower rates of co-morbidities [62].

One of the strengths of our study is the inclusion of a large and representative sample of ethnically and clinically diverse patients with T2DM seeking treatments in Singapore. In addition, PS matching was performed to balance baseline characteristics of patients between treatment groups and to minimise bias when assessing treatment effect [63]. Several variables were also used in the identification of T2DM patients such as age at diagnosis and treatment in addition to diagnosis codes. Thus the risk of misclassification for T2DM was low considering our study findings are also consistent with those reported in published real-world studies and clinical trials. There are however some limitations with using prescribing data. Prescribing data does not reflect actual ingestion and adherence to therapy but prescriptions indicated as cancelled or discontinued were excluded from the analyses, to capture medication use more accurately. Residual confounding may still remain after PS matching. Future studies with larger sample sizes or longer follow-up period may be required to further assess the effect of SGLT2 inhibitors by ethnicity on outcomes such as diabetic nephropathy. Possible switching between SGLT2 inhibitors and DPP4 inhibitors after treatment initiation was not accounted for. Finally, the benefits of SGLT2 inhibitors were potentially underestimated as reductions in body weight and blood pressure could not be assessed due to limitations of the database.

\section{Conclusions}

In summary, the results of our study showed that SGLT2 inhibitors were associated with improvements in glycaemic control and reduced risk of hospitalisations and deaths in patients with T2DM managed in the public healthcare setting in Singapore, and were well tolerated. However, such benefits were mostly observed in patients of Chinese ethnicity. Therefore, future studies should consider ethnicity as a key factor in overall disease management and the risk of developing T2DM-related complications. 


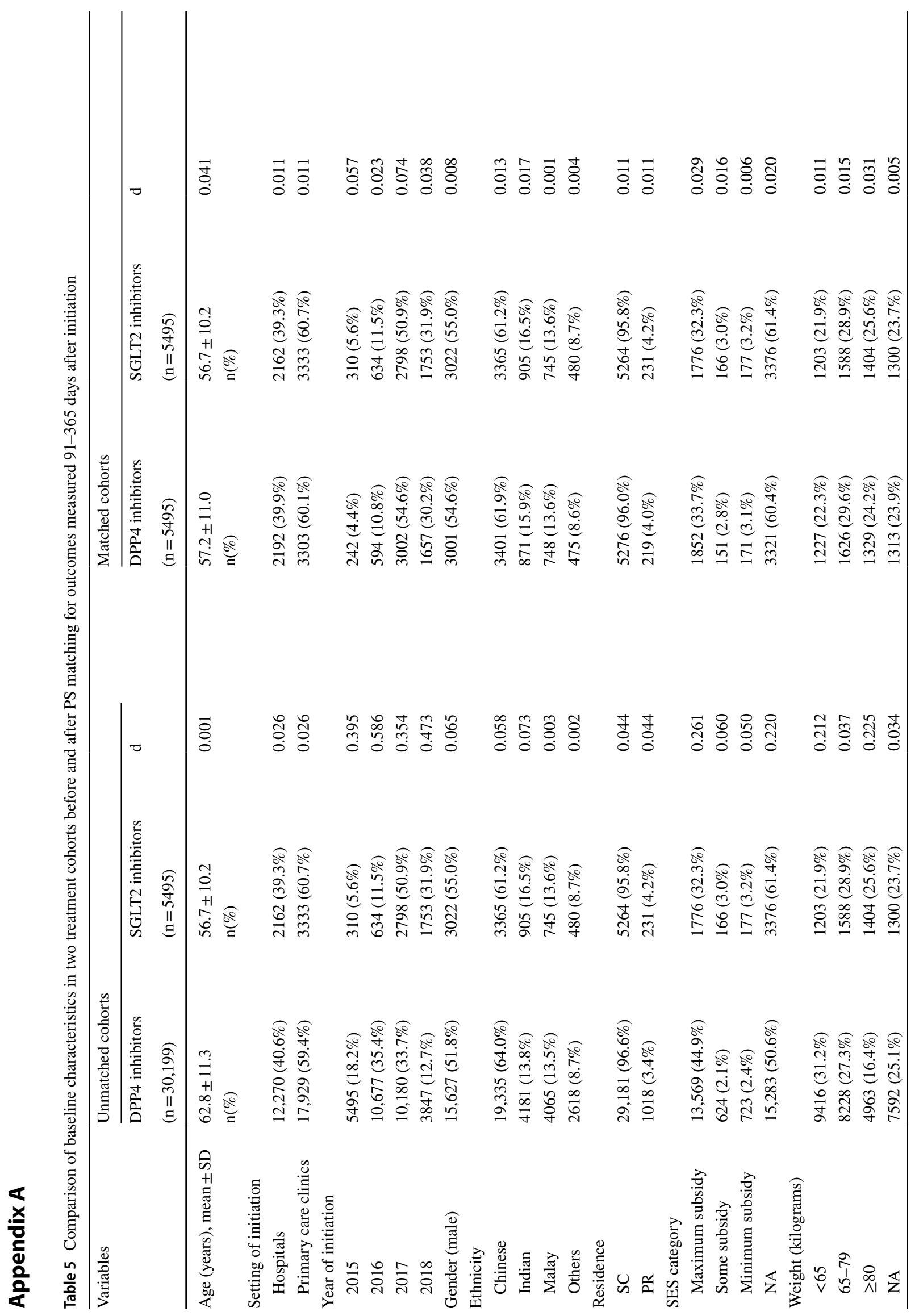




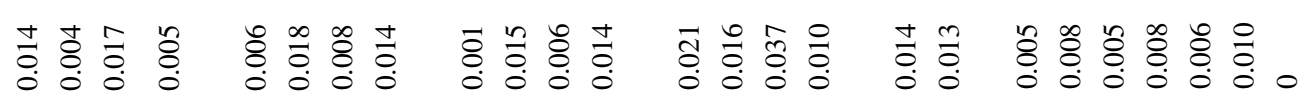

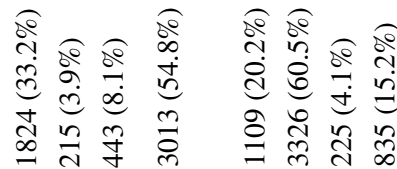

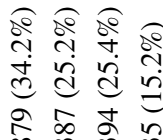

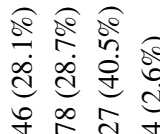

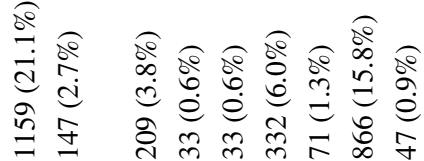

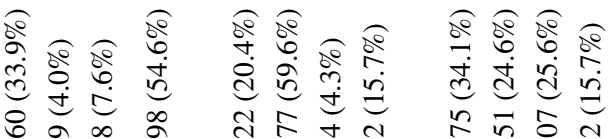

ते के के के

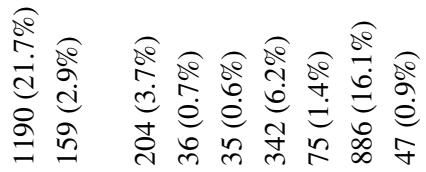

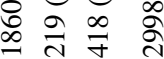

సิ

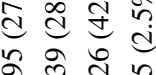

年令

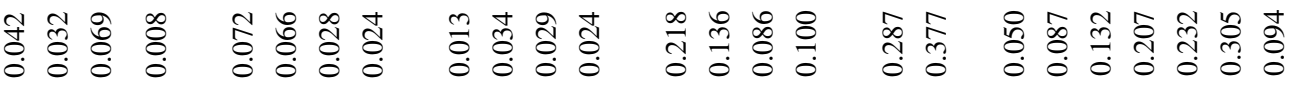

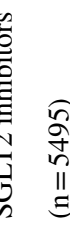

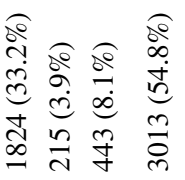

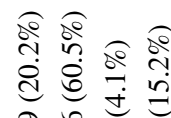

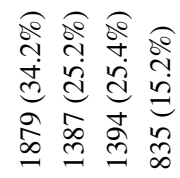

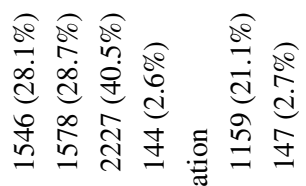

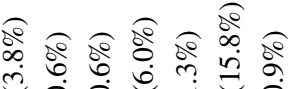

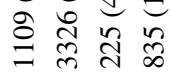

穴 $m$ m $m$ m

苛

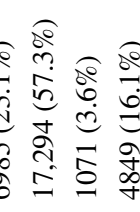

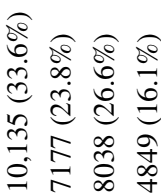

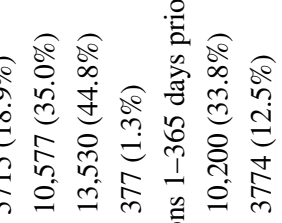

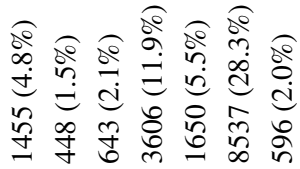

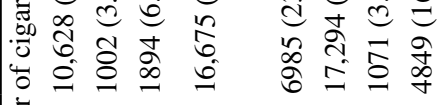

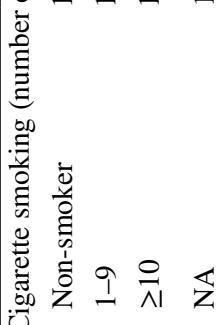

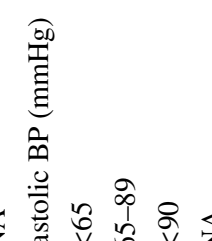

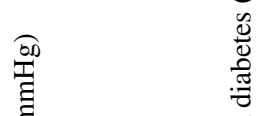

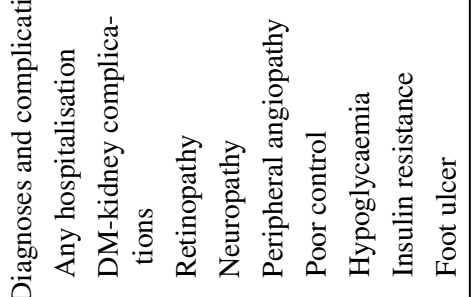




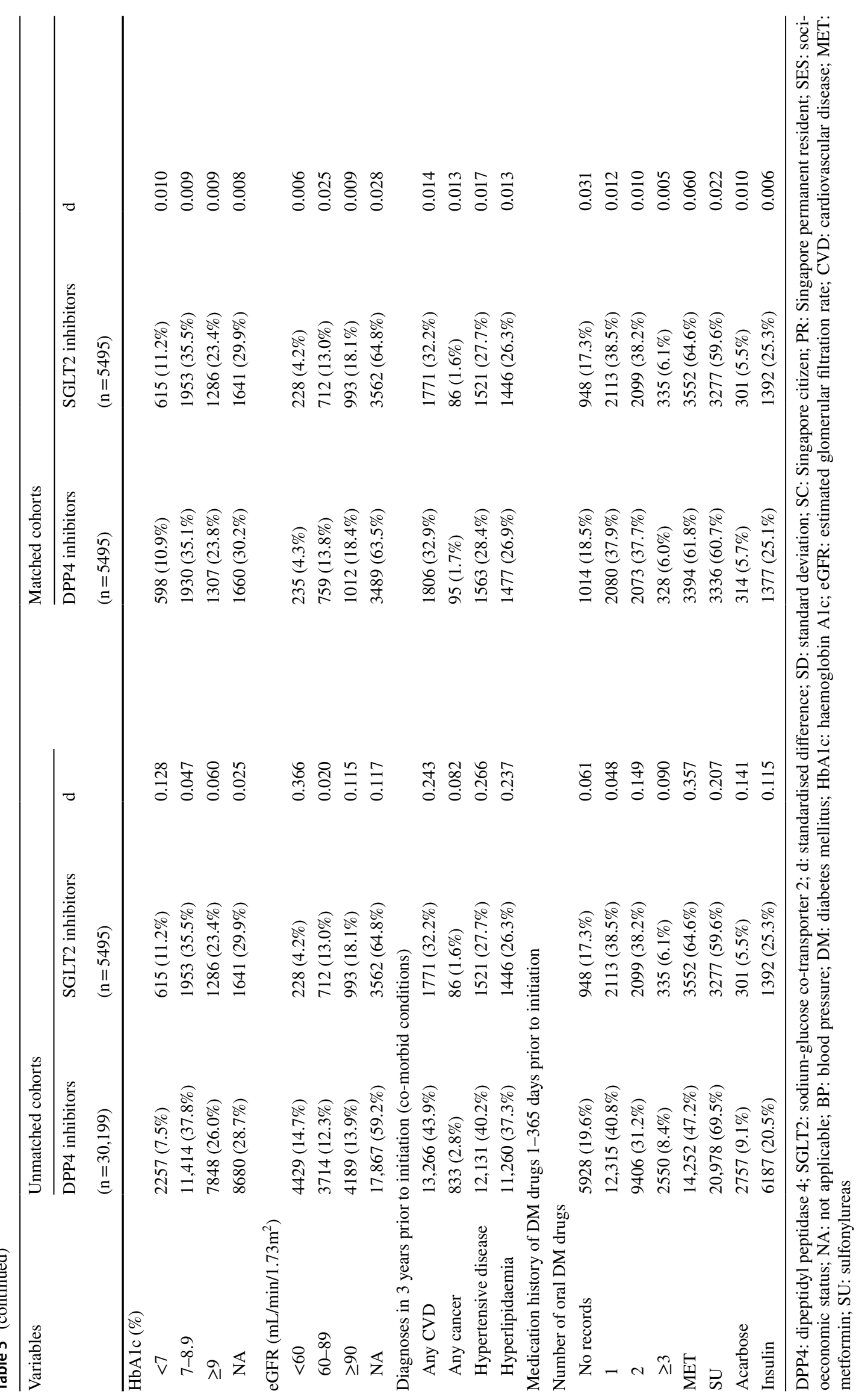


Fig. 2 Selection of study population for outcomes measured 91-365 days after initiation

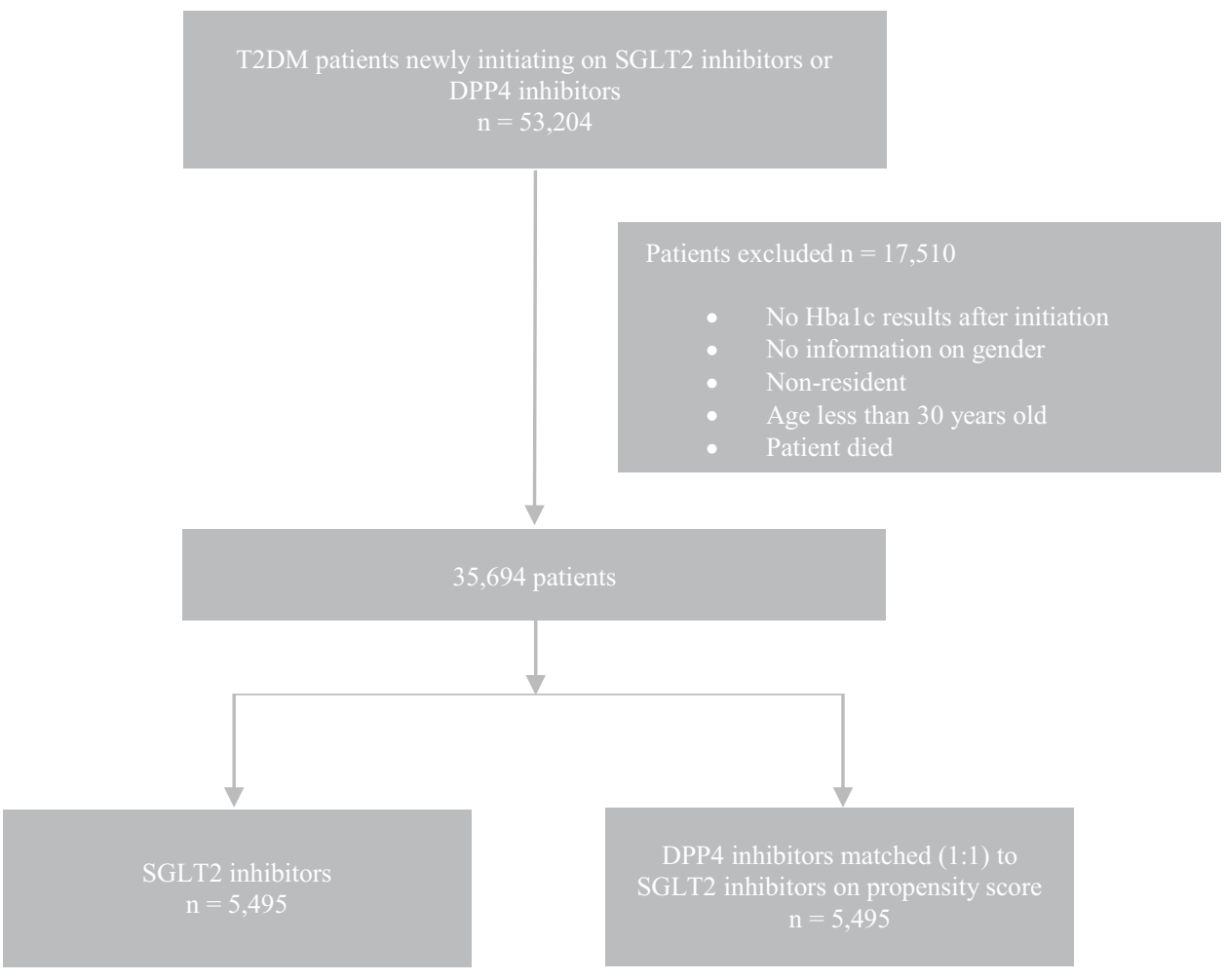




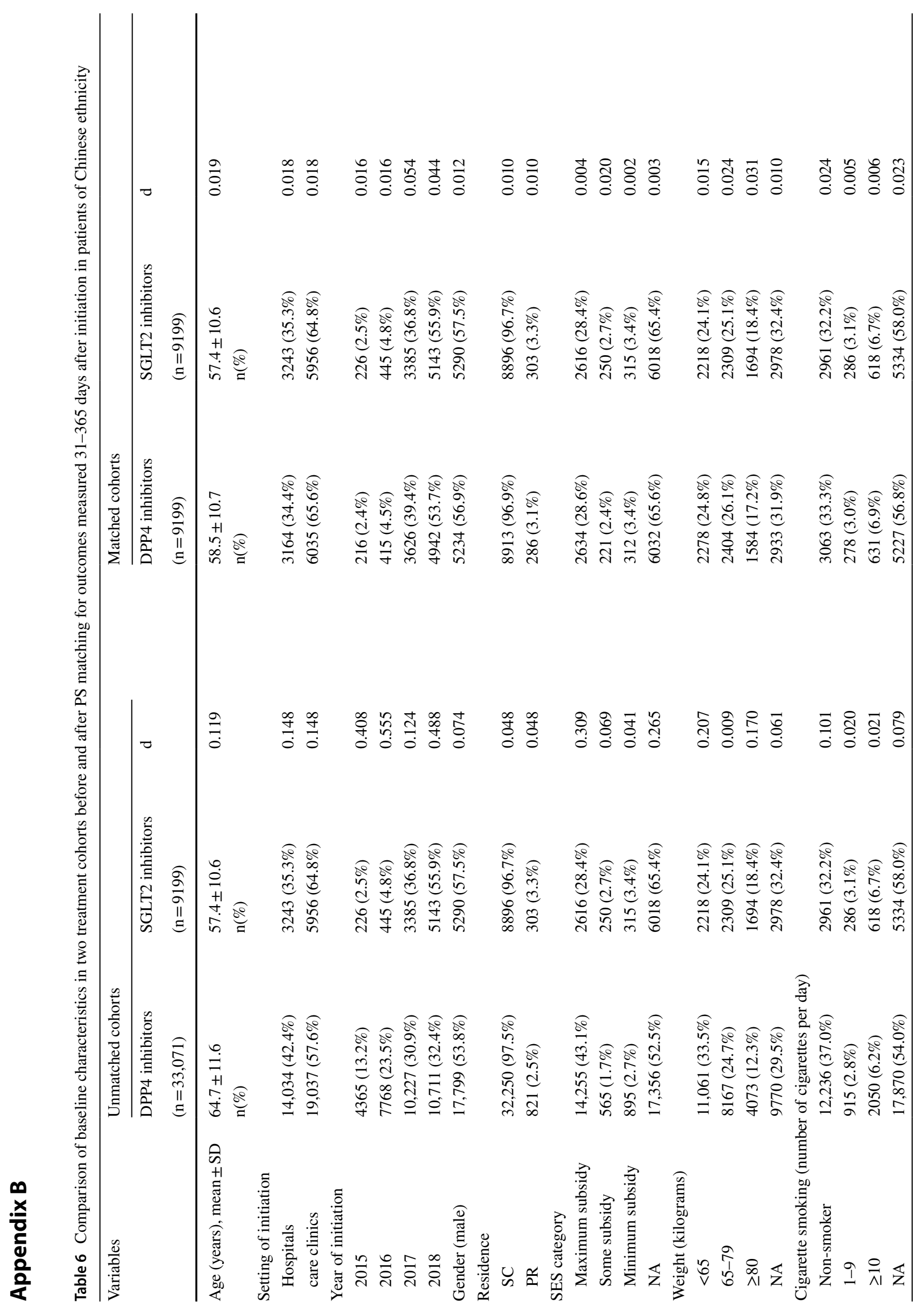




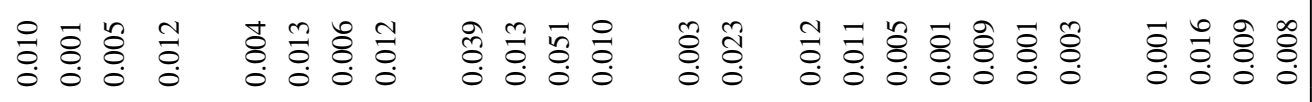

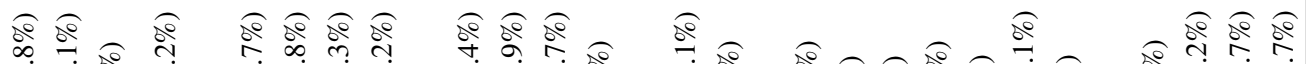

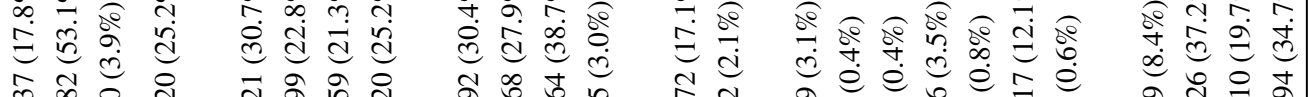

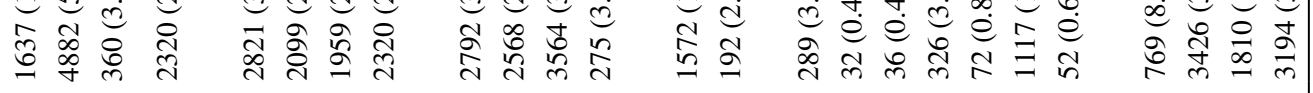

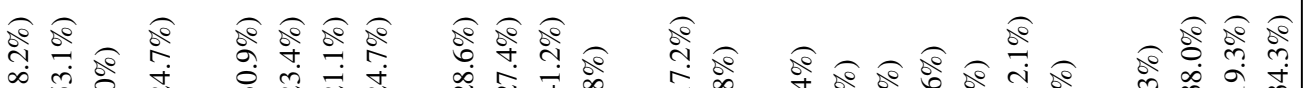

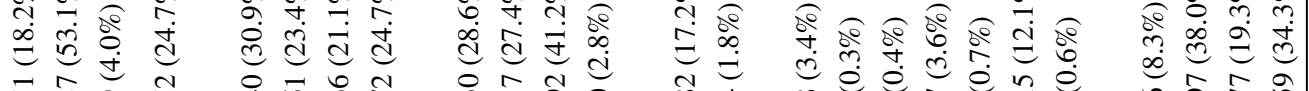

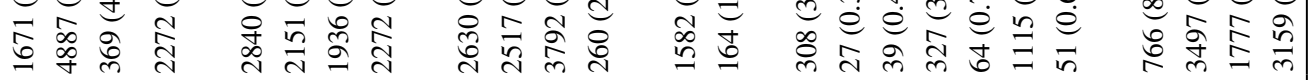

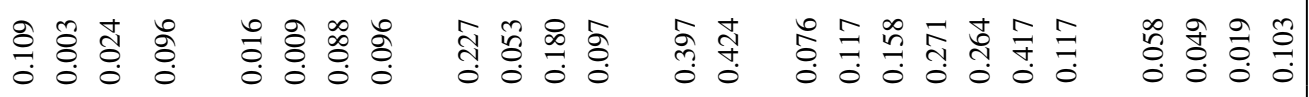

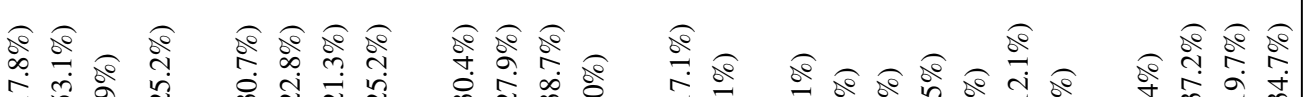

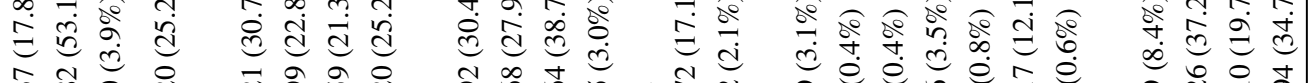

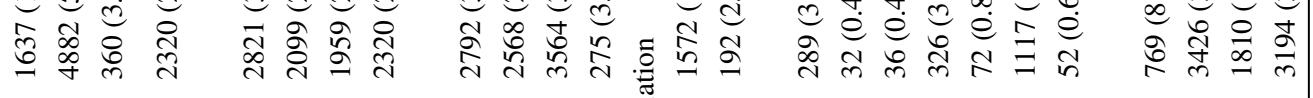
湆

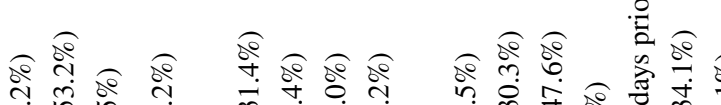
ते हैं

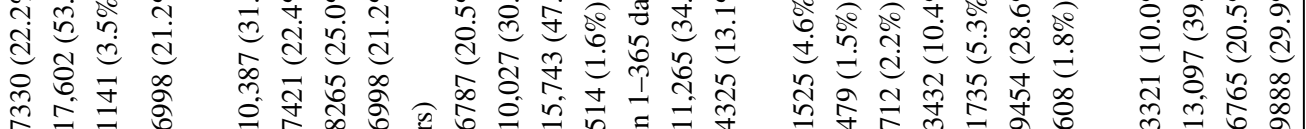

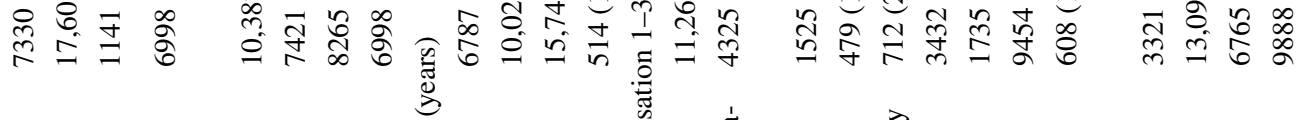

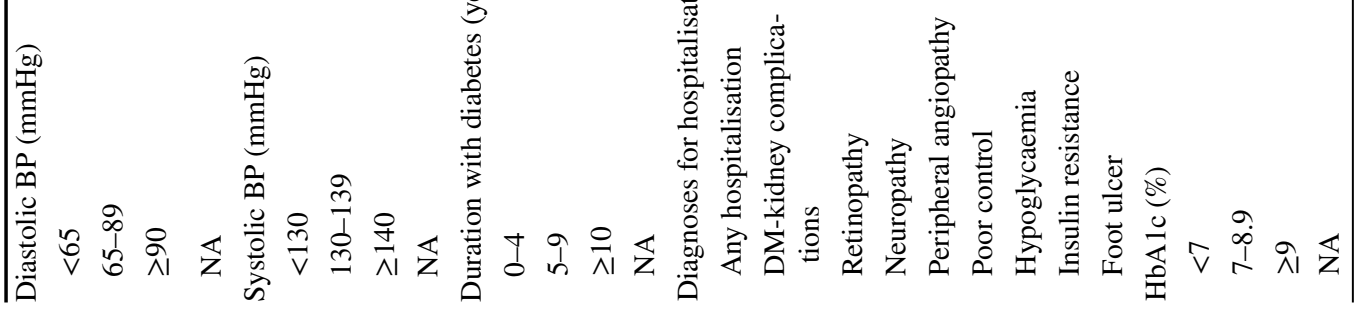




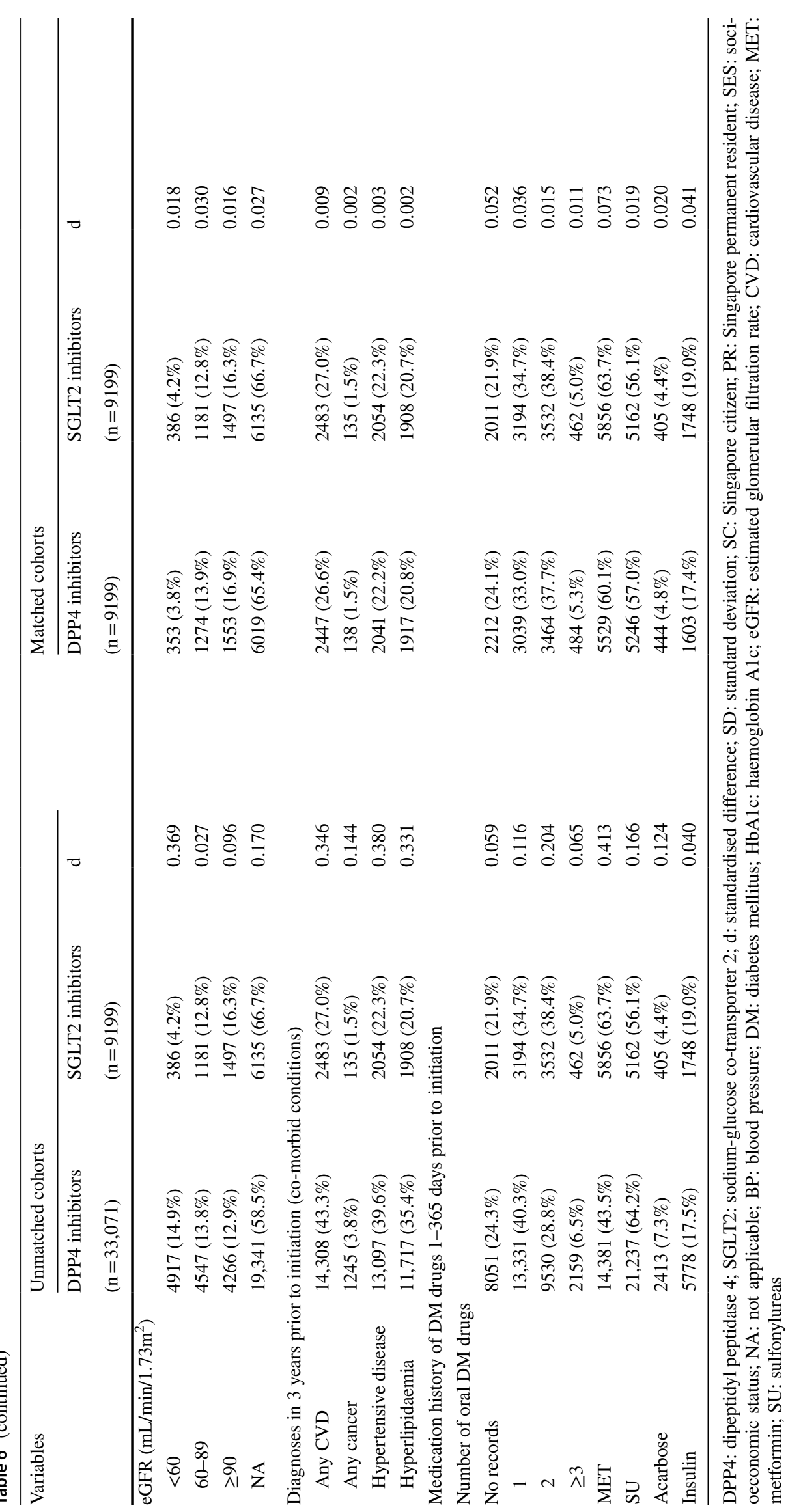




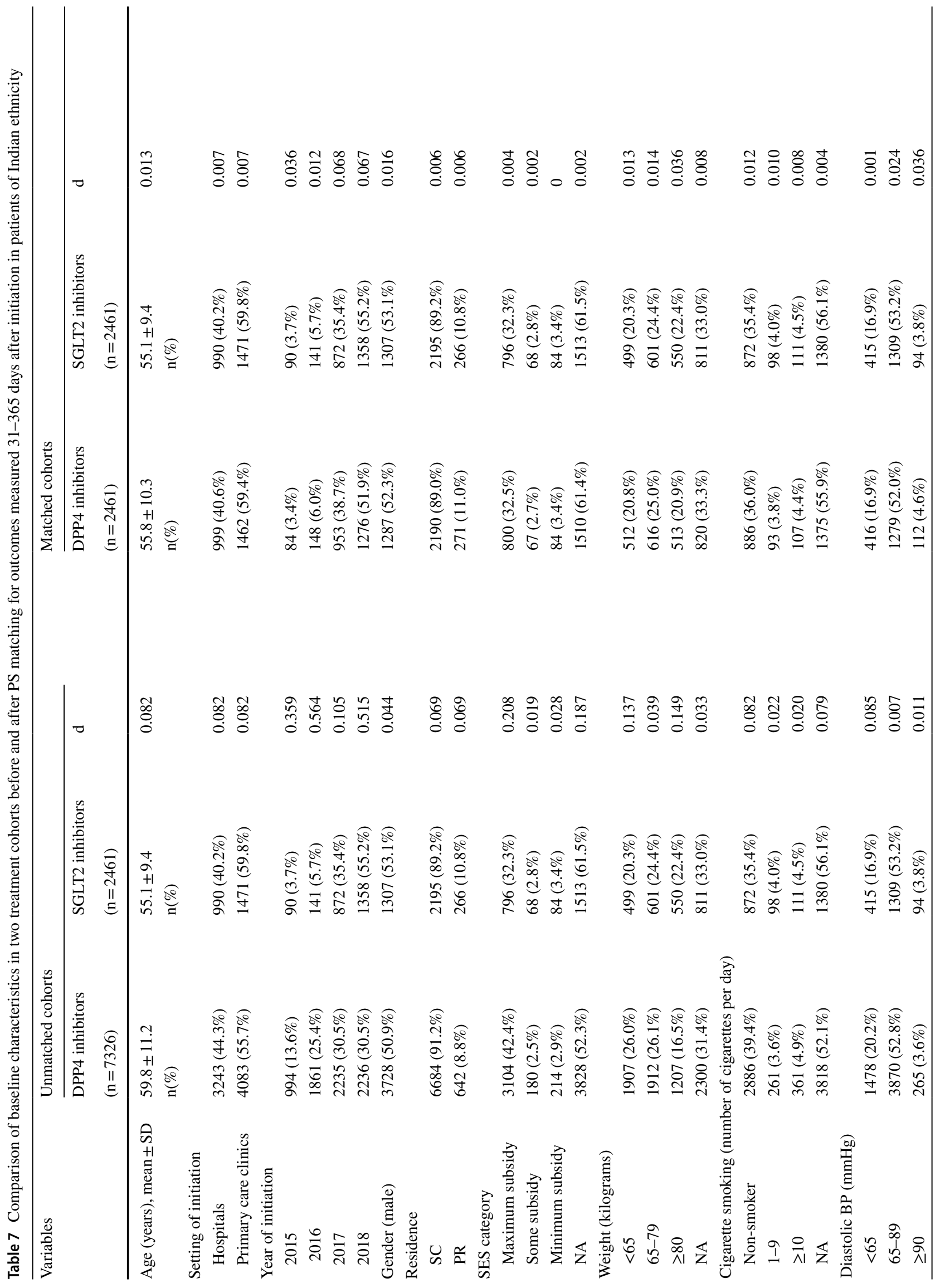




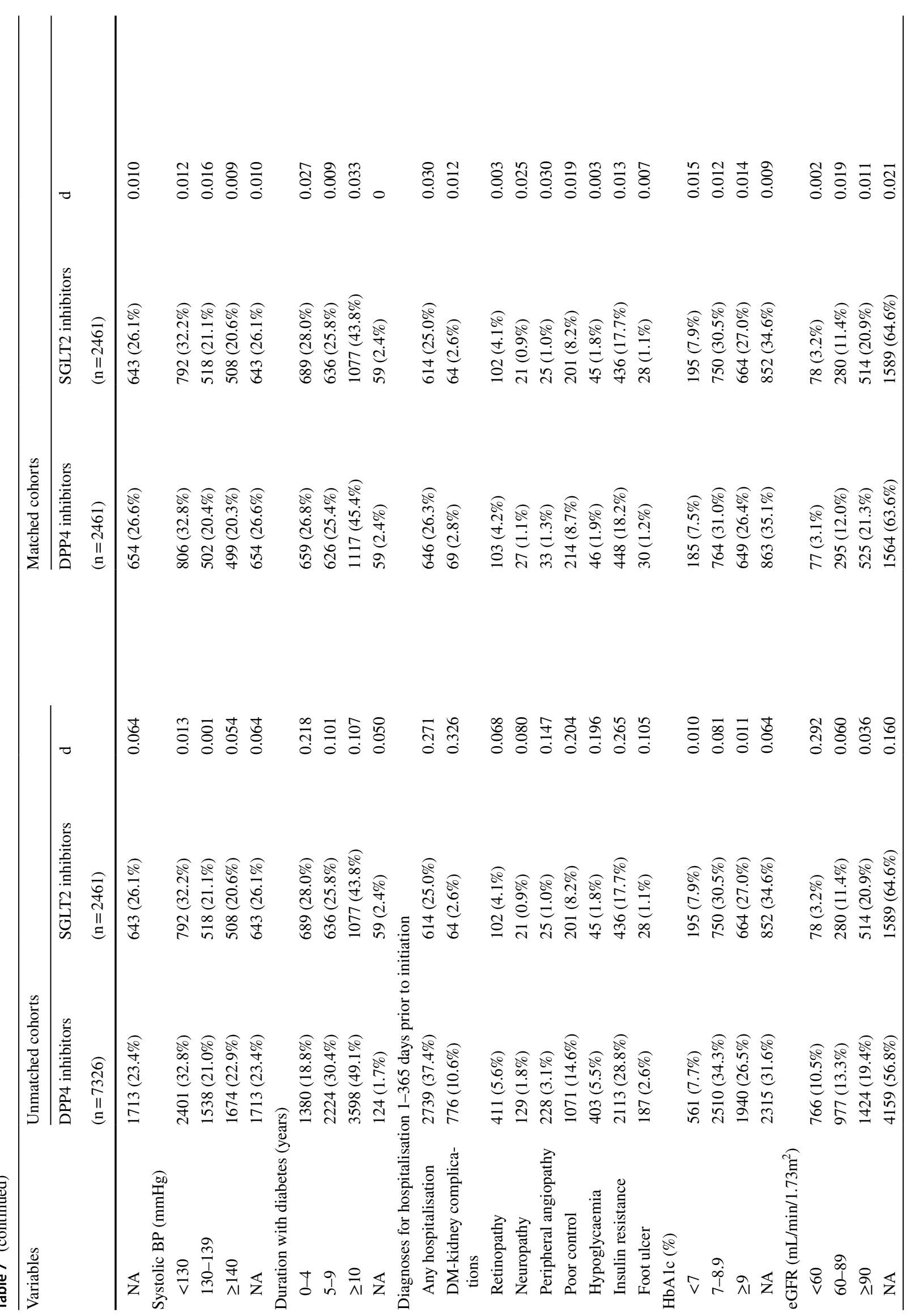




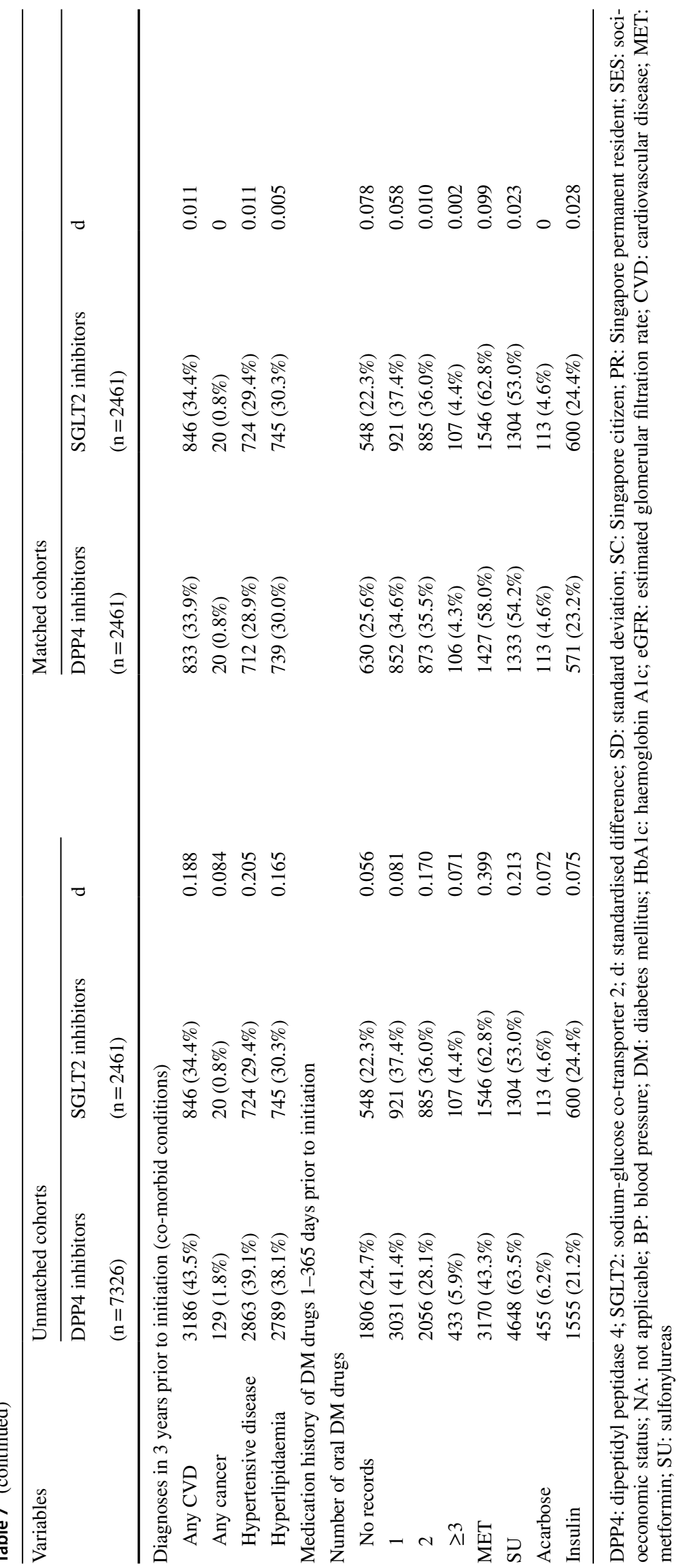




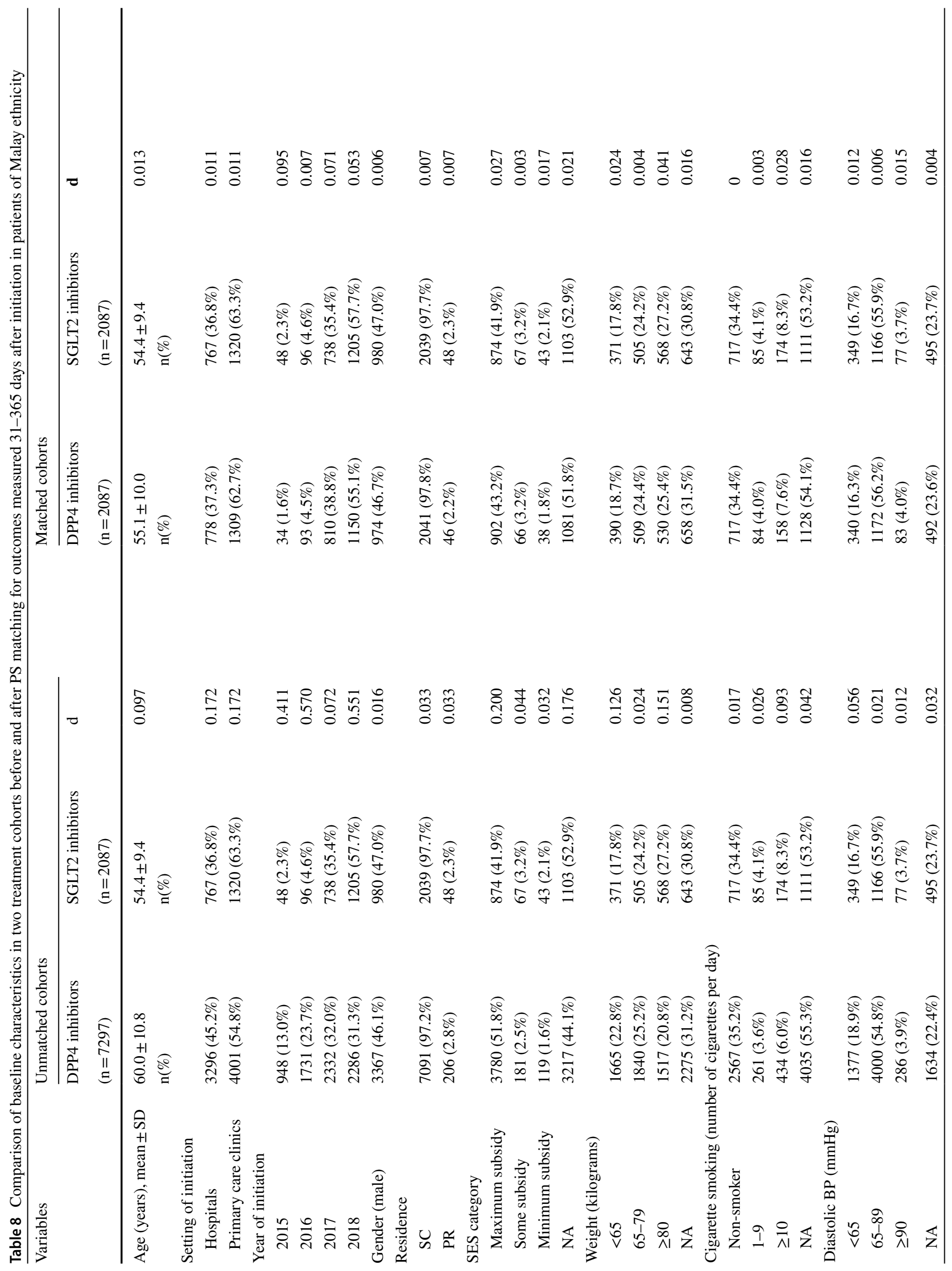




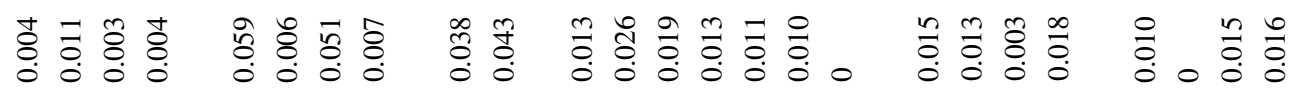

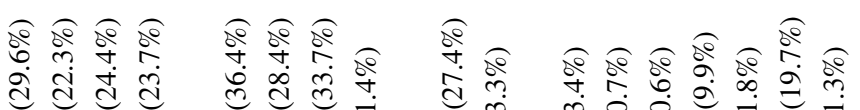

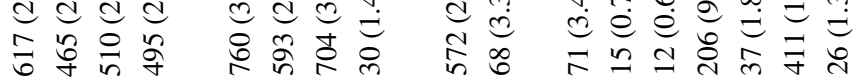

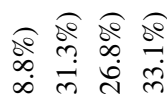

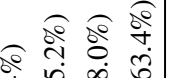

赵

范 :

$\therefore \stackrel{\infty}{\infty} \stackrel{n}{m}$

बृ 80

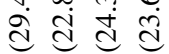

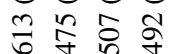

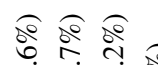

लृ

se

高哭

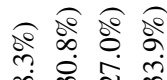

œ

咅

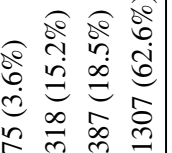

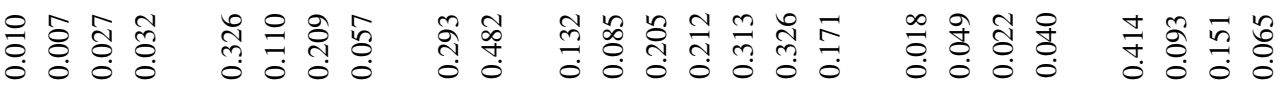

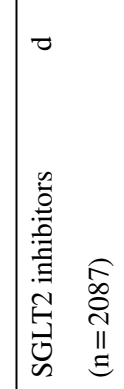

की 8 of

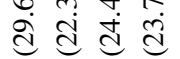

वृ $\frac{a}{2}$

के

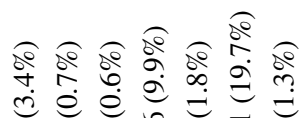

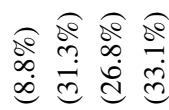

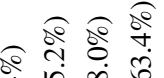

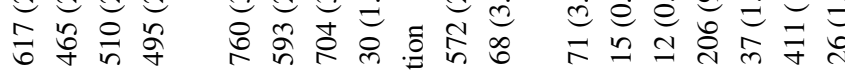

范 8 \&

$+\stackrel{\infty}{=}=$

강 $\underset{\infty}{\infty} \stackrel{\infty}{m}$

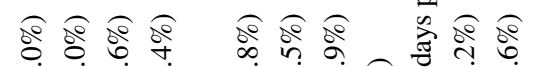

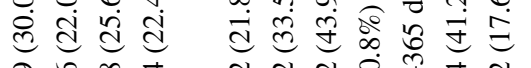

के

i d 0

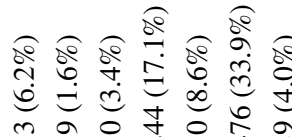

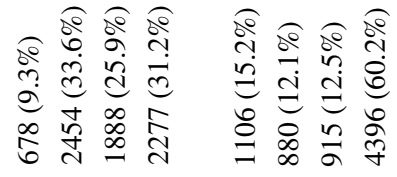

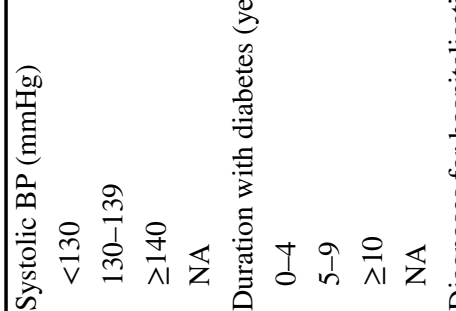

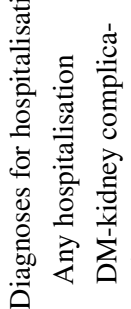

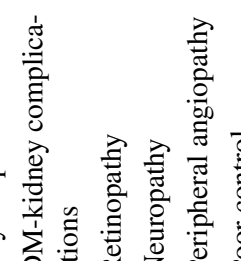

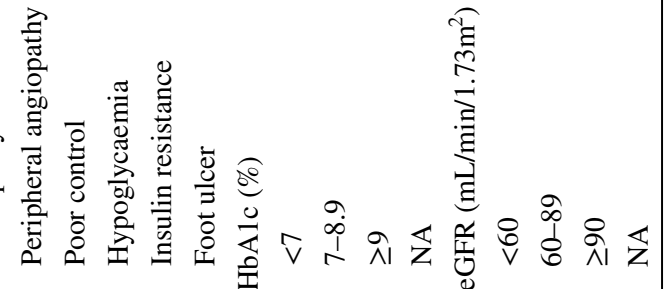




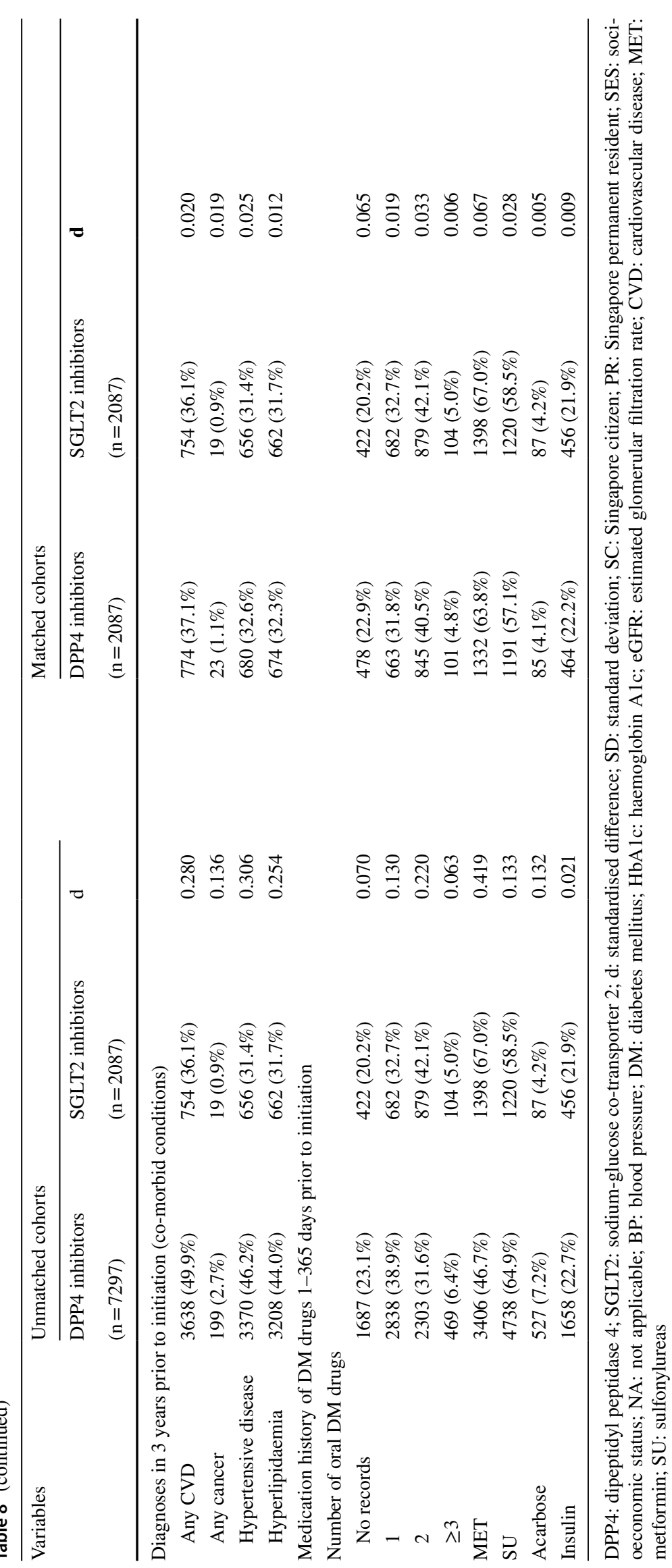




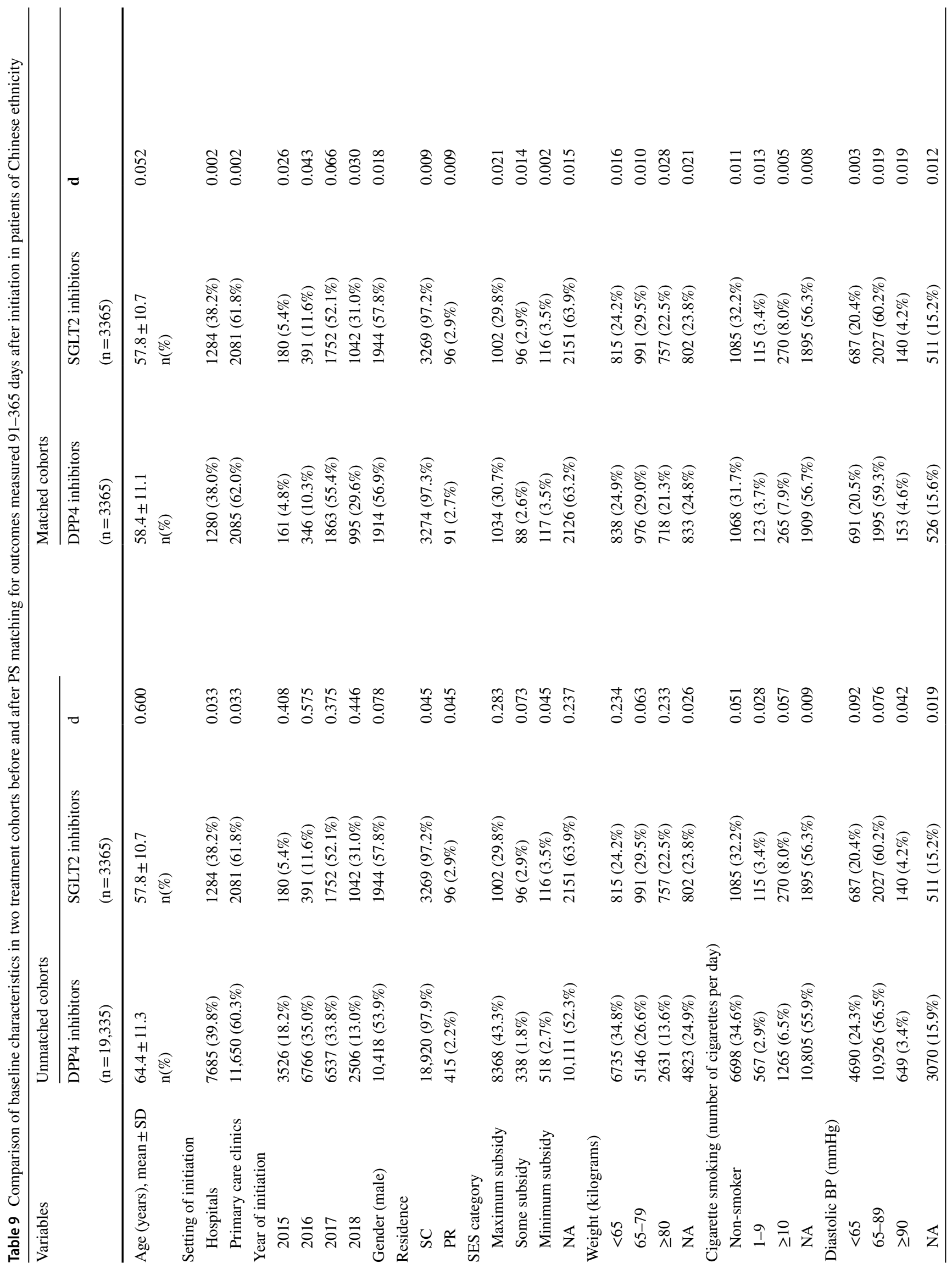




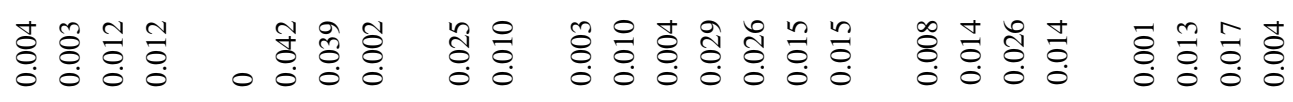

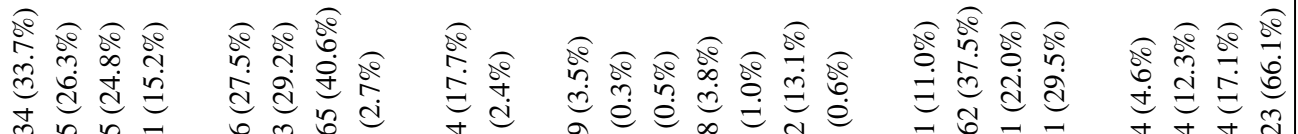

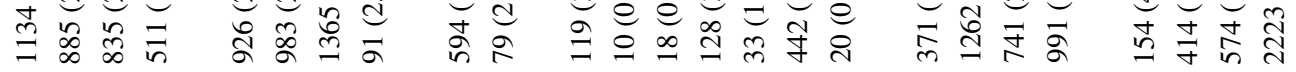

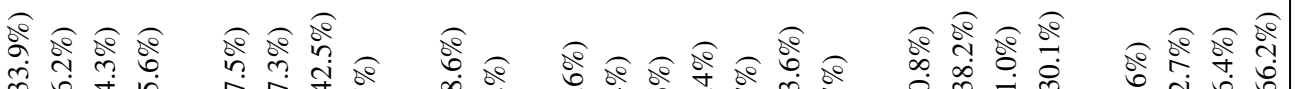

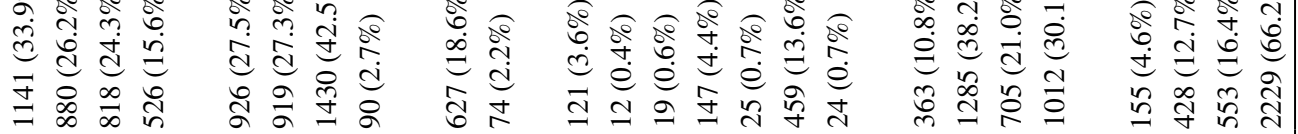

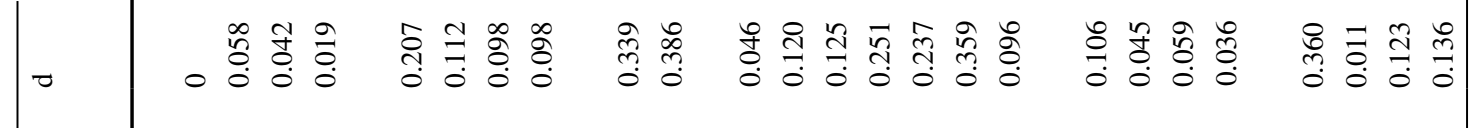

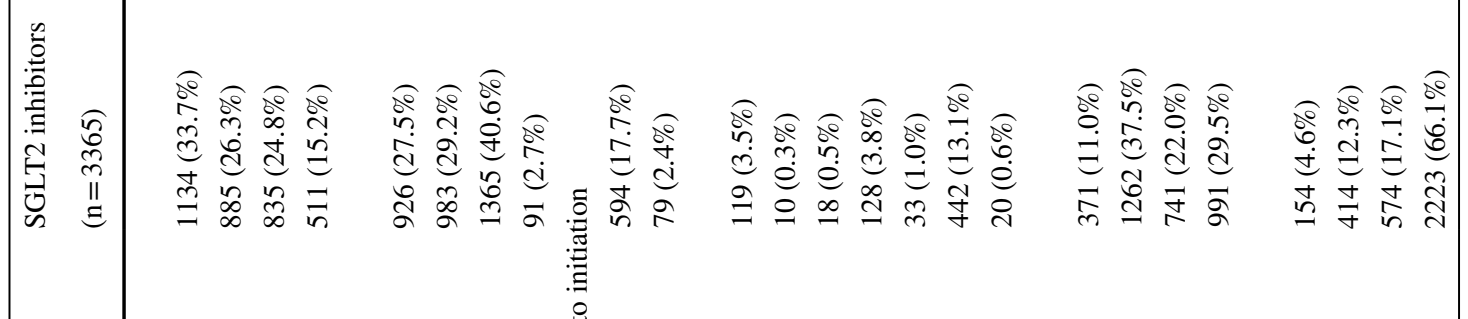

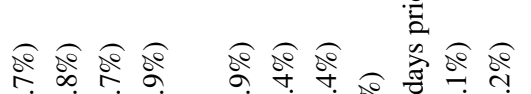

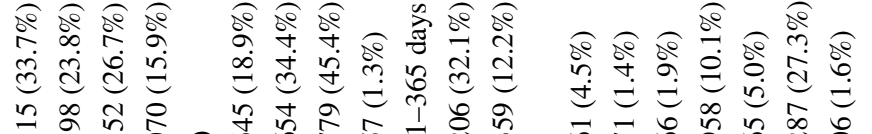

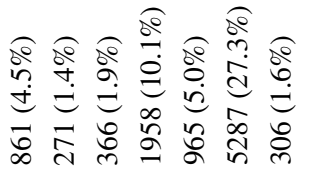

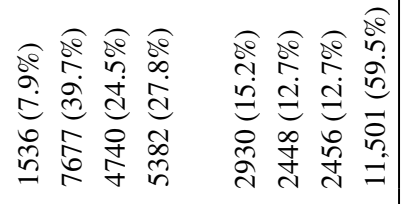
.
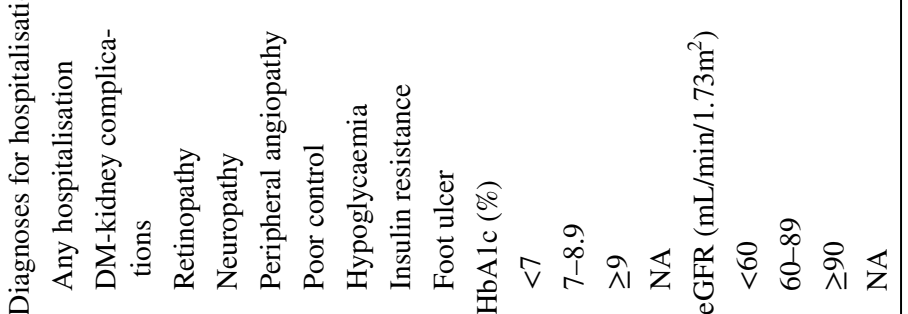


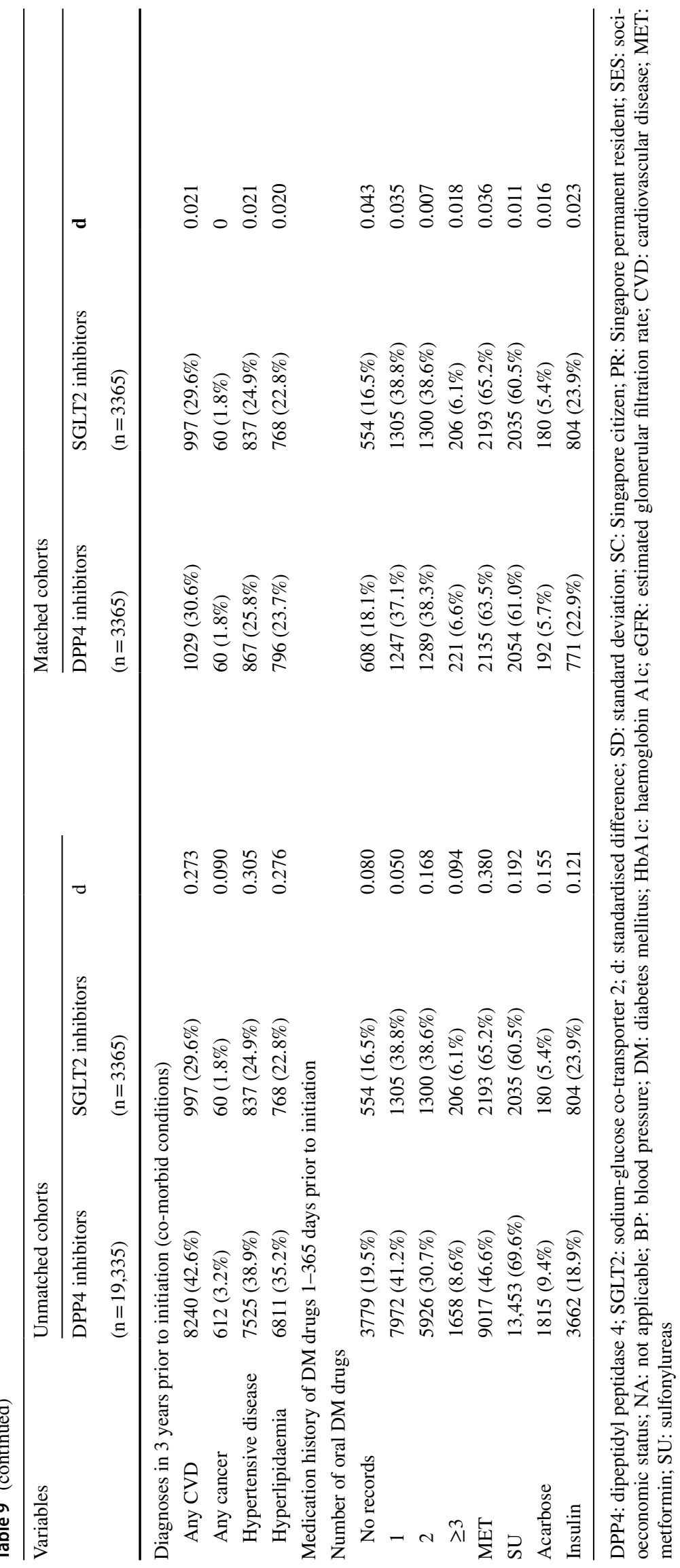




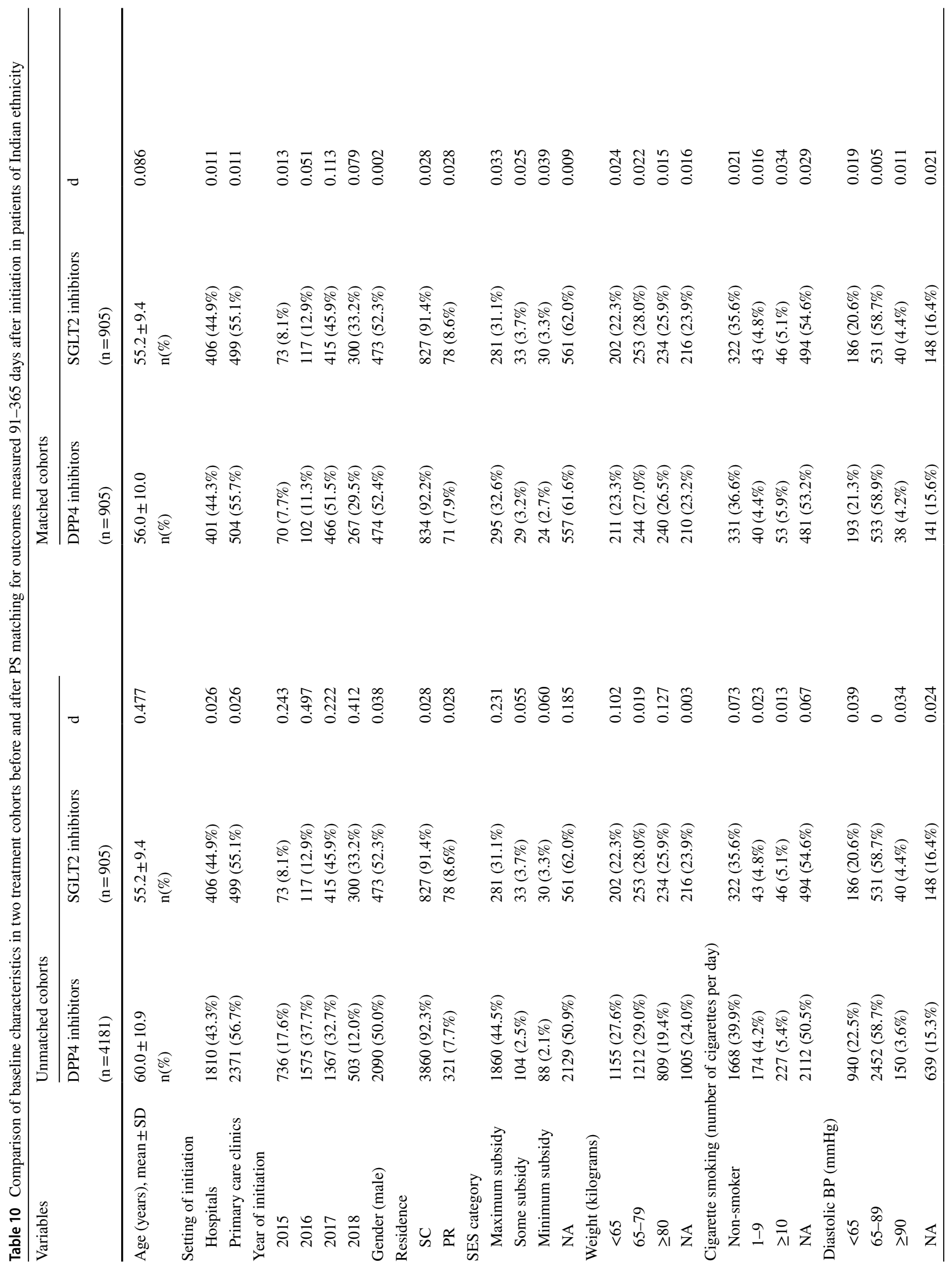




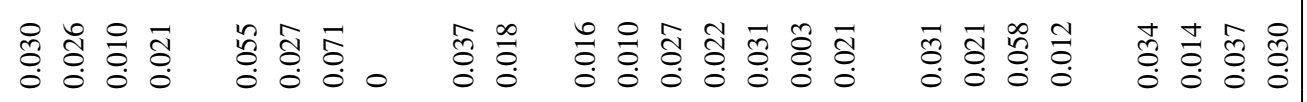

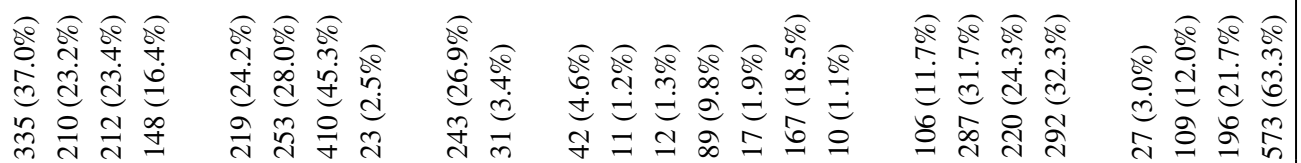

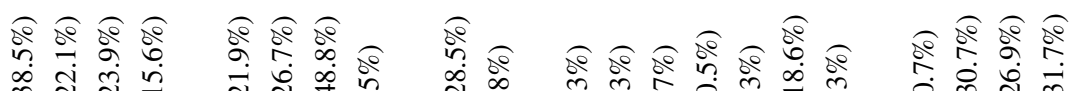

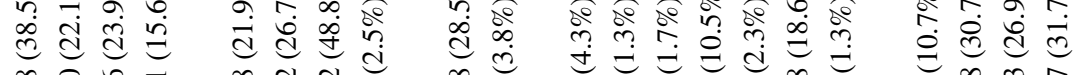

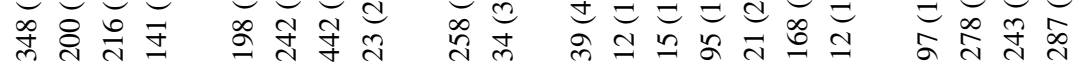

o in

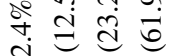

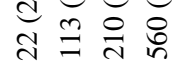

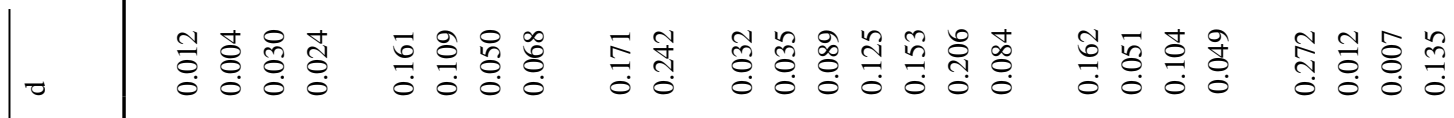

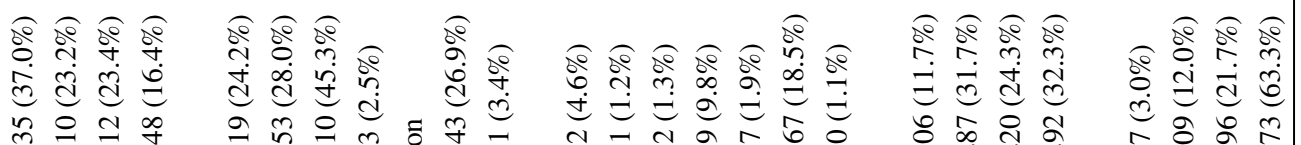

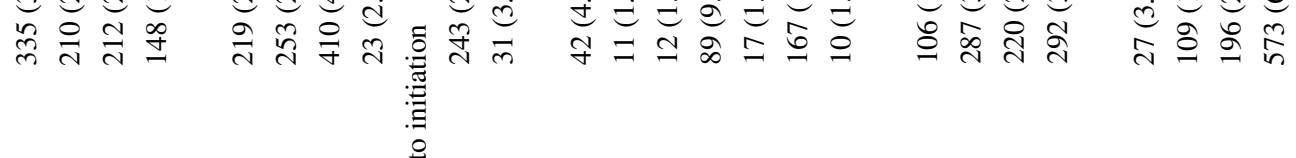

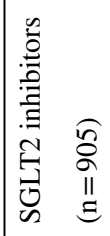

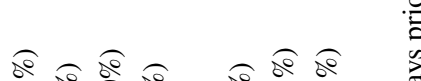
苛

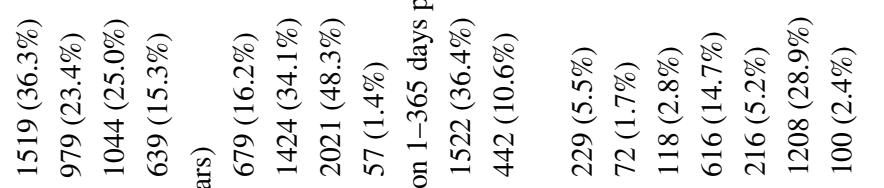

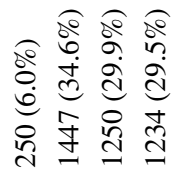
so 仓记

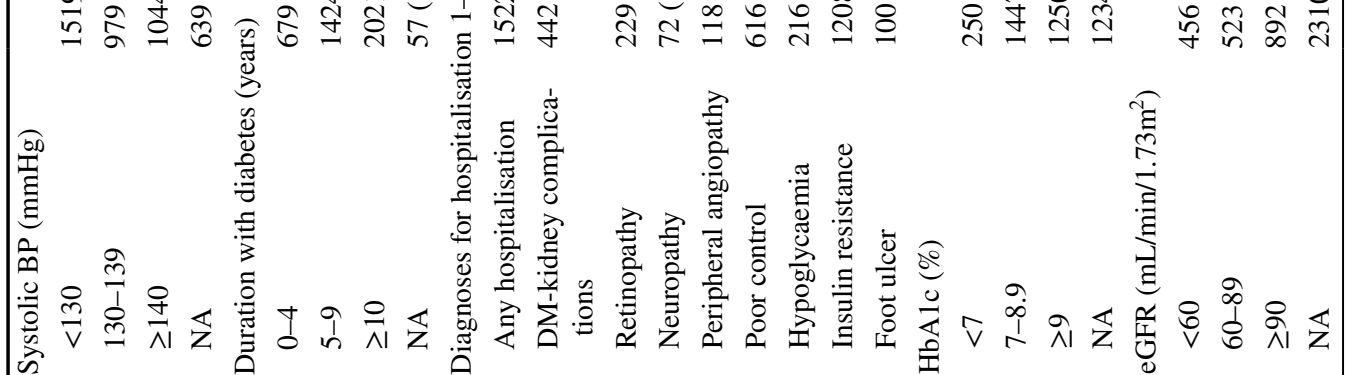




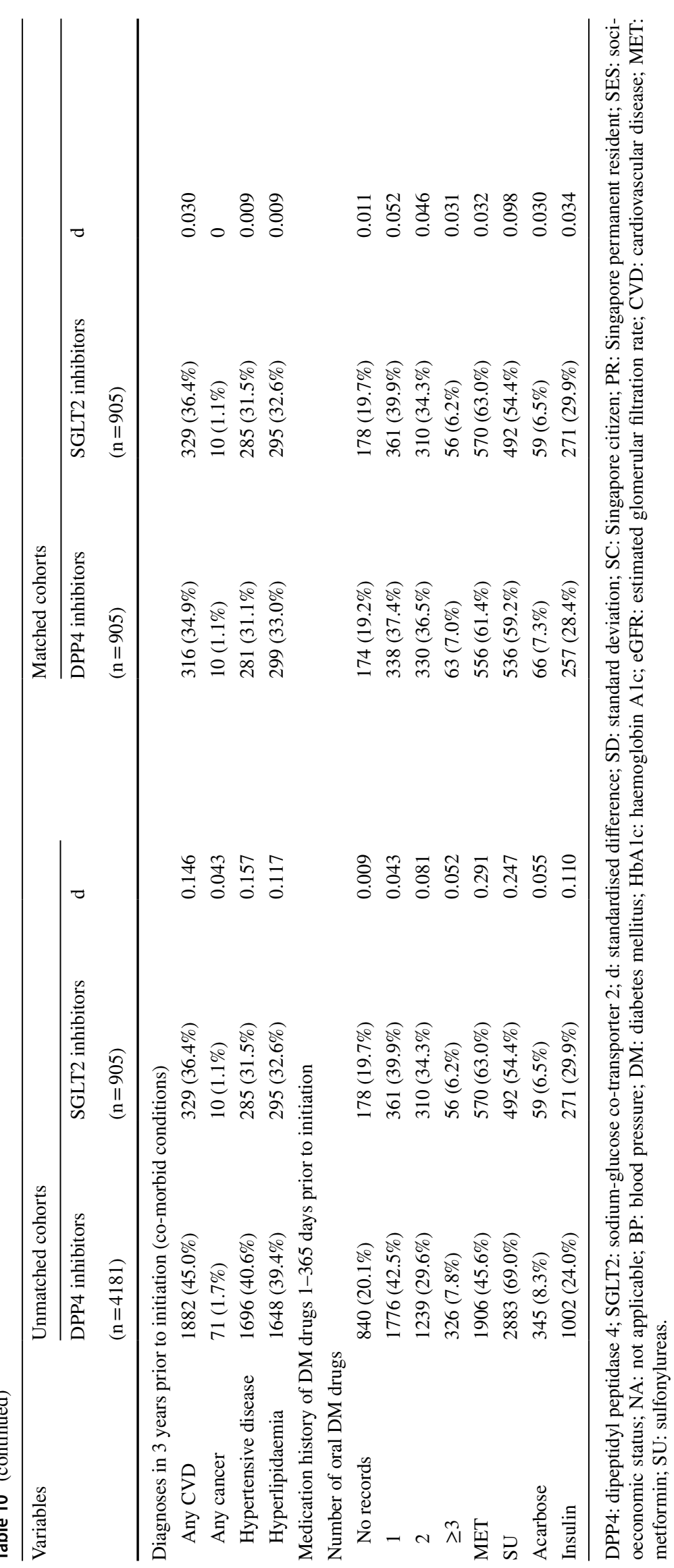




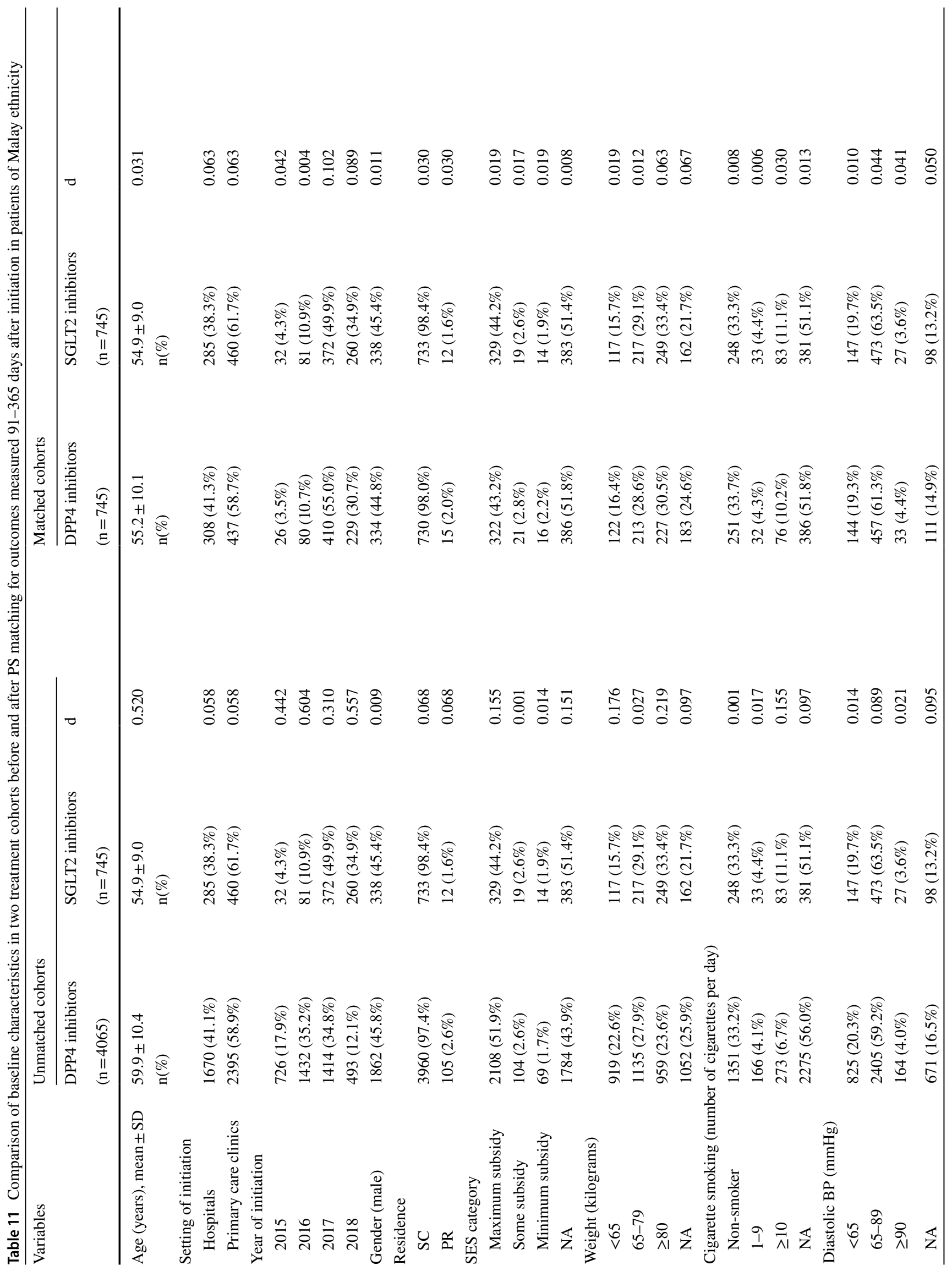




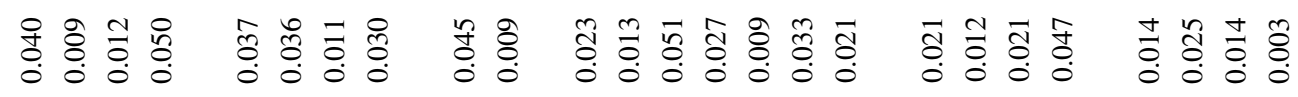

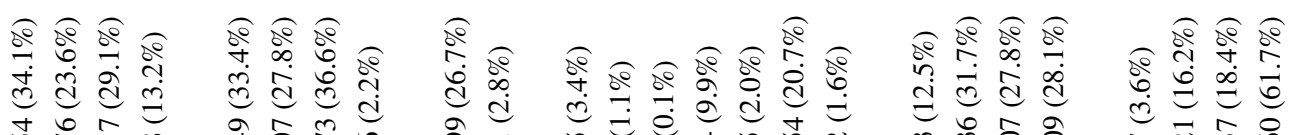

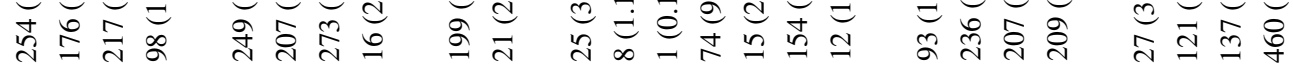

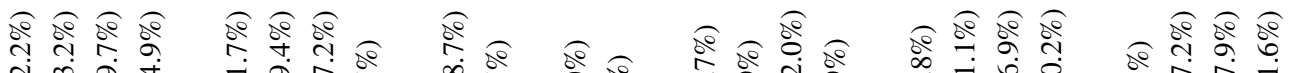

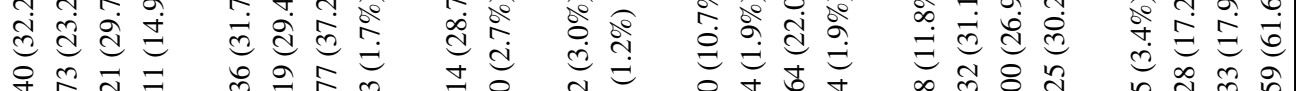

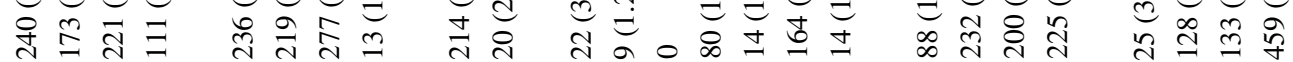

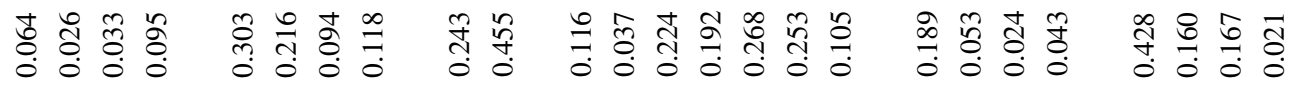

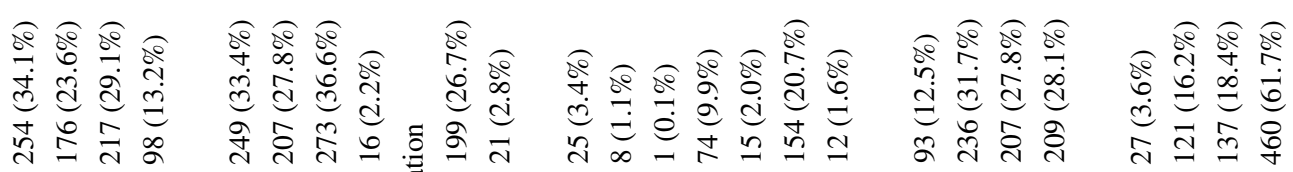

\section{(2)}

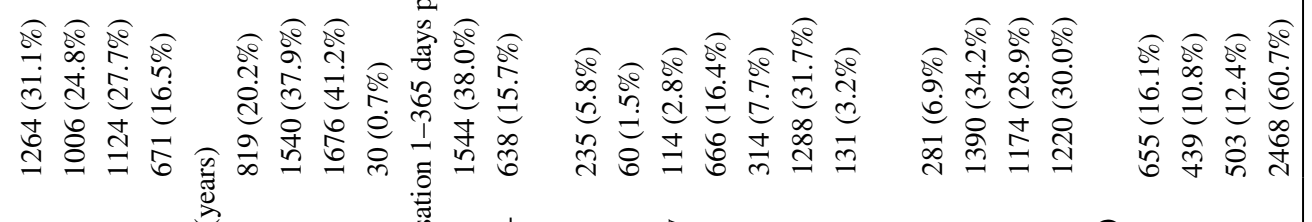




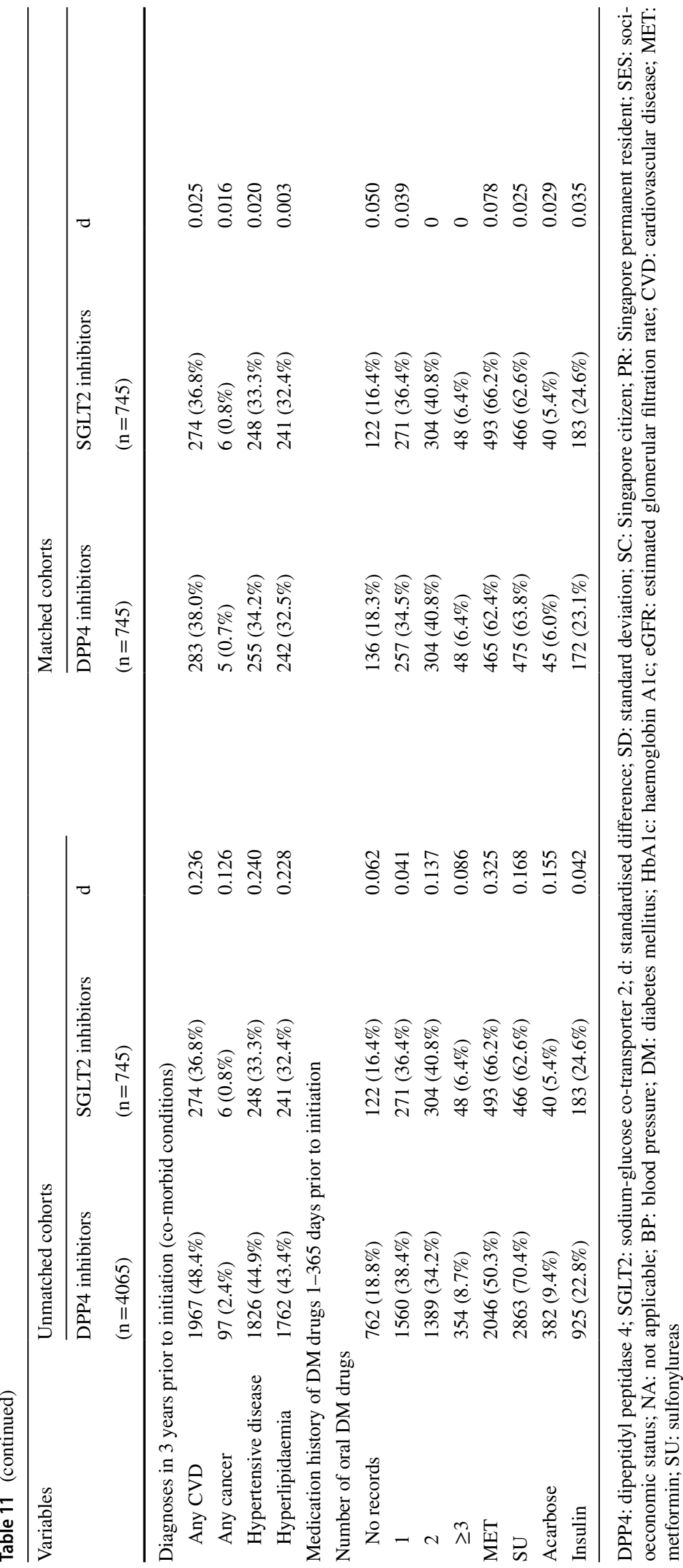


Abbreviations HbA1c: haemoglobin A1c; eGFR: estimated glomerular filtration rate; BP: blood pressure; SGLT2: sodium-glucose co-transporter 2; DPP4: dipeptidyl peptidase 4; MET: metformin; SU: sulfonylureas; T2DM: type 2 diabetes mellitus; T1DM: type 1 diabetes mellitus; DM: diabetes mellitus; CKD: chronic kidney disease; DKA: diabetic ketoacidosis; CVD: cardiovascular disease; HF: heart failure; UTI: urinary tract infection; SC: Singapore citizen; PR: Singapore permanent resident; SES: socioeconomic status; MOH: Ministry of Health, Singapore; ICD-10 AM: International Classification of Diseases, Tenth Revision Australian Modification; RR: risk ratio; CI: confidence interval; PS: propensity score; d: standardised difference; SD: standard deviation; MD: mean difference; HR: hazard ratio; OR: odds ratio; RCTs: randomised controlled trials; CVDREAL: Comparative Effectiveness of Cardiovascular Outcomes in New Users of Sodium-Glucose Cotransporter-2 Inhibitors; CANVAS: Canagliflozin Cardiovascular Assessment Study; EMPA-REG OUTCOME: Empagliflozin Cardiovascular Outcome Event Trial in Type 2 Diabetes Mellitus Patients-Removing Excess Glucose; UK: United Kingdom; US: United States; NA: not applicable

Authors' contributions All authors contributed to the conception, design of the study, interpretation of data, and read and approved the final manuscript. LGHG and JS analysed the data.

Data availability Data will not be publicly shared but is available on reasonable request and if legal implications are fulfilled.

\section{Declarations}

Ethics approval and consent to participate Ethics approval was not required as the intent of this study was to assess the effect of SGLT2 inhibitors on clinical outcomes for the purpose of improving routine clinical care.

\section{Consent for publication Not applicable.}

Competing interests The authors declare that they have no competing interests.

Open Access This article is licensed under a Creative Commons Attribution 4.0 International License, which permits use, sharing, adaptation, distribution and reproduction in any medium or format, as long as you give appropriate credit to the original author(s) and the source, provide a link to the Creative Commons licence, and indicate if changes were made. The images or other third party material in this article are included in the article's Creative Commons licence, unless indicated otherwise in a credit line to the material. If material is not included in the article's Creative Commons licence and your intended use is not permitted by statutory regulation or exceeds the permitted use, you will need to obtain permission directly from the copyright holder. To view a copy of this licence, visit http://creativecommons.org/licenses/by/4.0/.

\section{References}

1. Zimmet PZ, Magliano DJ, Herman WH, Shaw JE. Diabetes: a 21st century challenge. Lancet Diabetes Endocrinol. 2014;2(1):56-64.

2. International Diabetes Federation. IDF Diabetes Atlas, 9th edition. 2019.
3. Phan TP, Alkema L, Tai ES, Tan KHX, Yang Q, Lim W-Y, et al. Forecasting the burden of type 2 diabetes in Singapore using a demographic epidemiological model of Singapore. BMJ Open Diabetes Res Care. 2014;2:e000012.

4. World Health Organization. Global report on diabetes. 2016.

5. Schirr-Bonnans S, Costa N, Derumeaux-Burel H, Bos J, Lepage B, Garnault V, et al. Cost of diabetic eye, renal and foot complications: a methodological review. Eur J Health Econ. 2017;18(3):293-312.

6. Stetson B, Minges KE, Richardson CR. New directions for diabetes prevention and management in behavioral medicine. J Behav Med. 2017;40:127-44.

7. Yeung RO, Zhang Y, Luk A, Yang W, Sobrepena L, Yoon KH, et al. Metabolic profiles and treatment gaps in young-onset type 2 diabetes in Asia (the JADE programme): a cross-sectional study of a prospective cohort. Lancet Diabetes Endocrinol. 2014;2(12):935-43.

8. Ali MK, Bullard KM, Saaddine JB, Cowie CC, Imperatore G, Gregg EW. Achievement of goals in U.S. diabetes care, 19992010. N Engl J Med. 2013;368:1613-24.

9. Chaudhury A, Duvoor C, Reddy Dendi VS, Kraleti S, Chada A, Ravilla R, et al. Clinical review of antidiabetic drugs: implications for type 2 diabetes mellitus management. Front Endocrinol. 2017;8(6).

10. Agency for Care Effectiveness, Ministry of Health. Oral glucoselowering agents in type 2 diabetes mellitus - an update Singapore. 2017. Available from: https://www.ace-hta.gov.sg/docs/defaultsource/acgs/oral-glucose-lowering-agents-in-t2dm-(updated-on-3august-2017).pdf?sfvrsn=d0e6aca0_2. Accessed 14 Apr 2021.

11. Kahn SE, Haffner SM, Heise MA, Herman WH, Holman RR, Jones NP, et al. Glycemic durability of rosiglitazone, metformin, or glyburide monotherapy. N Engl J Med. 2006;355:2427-43.

12. Shao S-C, Chang K-C, Lin S-J, Chien R-N, Hung M-J, Chan Y-Y, et al. Favourable pleiotropic effects of sodium glucose cotransporter 2 inhibitors: head-to-head comparisons with dipeptidyl peptidase- 4 inhibitors in type 2 diabetes patients. Cardiovasc Diabetol. 2020;19(17):1-11.

13. Kim Y-G, Jeon JY, Han SJ, Kim DJ, Lee K-W, Kim HJ. Sodiumglucose co-transporter-2 inhibitors and the risk of ketoacidosis in patients with type 2 diabetes mellitus: a nationwide populationbased cohort study. Diabetes Obes Metab. 2018;20:1852-8.

14. Scheen AJ. Pharmacokinetics and clinical use of incretin-based therapies in patients with chronic kidney disease and type 2 diabetes. Clin Pharmacokinet. 2015;54(1):1-21.

15. Scheen AJ. Pharmacokinetics, pharmacodynamics and clinical use of SGLT2 inhibitors in patients with type 2 diabetes mellitus and chronic kidney disease. Clin Pharmacokinet. 2015;54(7):691-708.

16. Scheen AJ. Pharmacodynamics, efficacy and safety of sodiumglucose co-transporter type 2 (SGLT2) inhibitors for the treatment of type 2 diabetes mellitus. Drugs. 2015;75:33-59.

17. Agency for Care Effectiveness, Ministry of Health. Sodium-glucose co-transporter 2 (SGLT2) inhibitors for treating type 2 diabetes mellitus - technology guidance from the MOH Drug Advisory Committee Singapore. 2021. Available from: https://www.ace-hta. gov.sg/docs/default-source/drug-guidances/sodium-glucose-cotransporter-2-(sglt2)-inhibitors-for-treating-type-2-diabetes-melli tus-(updated-1-sept-2020).pdf?sfvrsn=694a3265_2. Accessed 15 Apr 2021.

18. Shao YL, Yee KH, Koh SK, Wong YF, Yeoh LY, Low S, et al. Short-term outcomes of patients with type 2 diabetes mellitus treated with canagliflozin compared with sitagliptin in a realworld setting. Singap Med J. 2018;59(5):251-6.

19. Ismail IS, Nazaimoon WM, Mohamad WB, Letchuman R, Singaraveloo M, Pendek R, et al. Sociodemographic determinants of glycaemic control in young diabetic patients in peninsular Malaysia. Diabetes Res Clin Pract. 2000;47(1):57-69. 
20. Huo L, Harding JL, Peeters A, Shaw JE, Magliano DJ. Life expectancy of type 1 diabetic patients during 1997-2010: a national Australian registry-based cohort study. Diabetologia. 2016;59:1177-85.

21. Menke A, Orchard TJ, Imperatore G, Bullard KM, Mayer-Davis E, Cowie CC. The prevalence of type 1 diabetes in the United States. Epidemiology. 2013;24(5):773-4.

22. Austin PC. Balance diagnostics for comparing the distribution of baseline covariates between treatment groups in propensity-score matched samples. Stat Med. 2009;28:3083-107.

23. Normand S-LT, Landrum MB, Guadagnoli E, Ayanian JZ, Ryan TJ, Cleary PD, et al. Validating recommendations for coronary angiography following acute myocardial infarction in the elderly: a matched analysis using propensity scores. J Clin Epidemiol. 2001;54:387-98

24. Zou G. A modified Poisson regression approach to prospective studies with binary data. Am J Epidemiol. 2004;159(7):702-6.

25. Woo V, Bell A, Clement M, Noronha L, Tsoukas MA, Camacho F, et al. CANadian CAnagliflozin REgistry: effectiveness and safety of canagliflozin in the treatment of type 2 diabetes mellitus in Canadian clinical practice. Diabetes Obes Metab. 2019;21:691-9.

26. Thayer S, Chow W, Korrer S, Aguilar R. Real-world evaluation of glycemic control among patients with type 2 diabetes mellitus treated with canagliflozin versus dipeptidyl peptidase- 4 inhibitors. Curr Med Res Opin. 2016;32(6):1087-96.

27. Buysman EK, Chow W, Henk HJ, Rupnow MFT. Characteristics and outcomes of patients with type 2 diabetes mellitus treated with canagliflozin: a real-world analysis. BMC Endocr Disord. 2015;15:67.

28. Huang H, Bell KF, Gani R, Tugwell CW, Eudicone JM, KrukasHampel MR. A retrospective real-world study of dapagliflozin versus other oral antidiabetic drugs added to metformin in patients with type 2 diabetes. Am J Mang Care. 2018;24:S132-S7.

29. Fadini GP, Zatti G, Baldi I, Bottigliengo D, Consoli A, Giaccari A, et al. Use and effectiveness of dapagliflozin in routine clinical practice: an Italian multicentre retrospective study. Diabetes Obes Metab. 2018;20:1781-6.

30. Morales C, Bellido V, Tejera C, Goñi F, Palomares R, Sevillano $\mathrm{C}$, et al. DAPA-RWE: a retrospective multicenter study comparing dapagliflozin and sitagliptin in patients with type 2 diabetes treated under routine clinical practice in Spain. J Comp Eff Res. 2021;10(10):815-21.

31. Schernthaner G, Gross JL, Rosenstock J, Guarisco M, Fu M, Yee $\mathrm{J}$, et al. Canagliflozin compared with sitagliptin for patients with type 2 diabetes who do not have adequate glycemic control with metformin plus sulfonylurea: a 52-week randomized trial. Diabetes Care. 2013;36:2508-15.

32. Lavalle-Gonzalez FJ, Januszewicz A, Davidson J, Tong C, Qiu R, Canovatchel W, et al. Efficacy and safety of canagliflozin compared with placebo and sitagliptin in patients with type 2 diabetes on background metformin monotherapy: a randomised trial. Diabetologia. 2013;56:2582-92.

33. Wang Z, Sun J, Han R, Fan D, Dong X, Luan Z, et al. Efficacy and safety of sodium-glucose cotransporter-2 inhibitors versus dipeptidyl peptidase- 4 inhibitors as monotherapy or add-on to metformin in patients with type 2 diabetes mellitus: a systematic review and meta-analysis. Diabetes Obes Metab. 2018;20:113-20.

34. Hong CY, Chia KS, Hughes K, Ling SL. Ethnic differences among Chinese, Malay and Indian patients with type 2 diabetes mellitus in Singapore. Singap Med J. 2004;45(4):154-60.

35. Yaghootkar H, Whitcher B, Bell JD, Thomas EL. Ethnic differences in adiposity and diabetes risk - insights from genetic studies. J Intern Med. 2020;288:271-83

36. Monami M, Nreu B, Zannoni S, Lualdi C, Mannucci E. Effects of SGLT-2 inhibitors on diabetic ketoacidosis: a meta-analysis of randomised controlled trials. Diabetes Res Clin Pract. 2017;130:53-60.

37. Qiu R, Balis D, Xie J, Davies MJ, Desai M, Meininger G. Longerterm safety and tolerability of canagliflozin in patients with type 2 diabetes: a pooled analysis. Curr Med Res Opin. 2017;33:553-62.

38. Zhang X-L, Zhu Q-Q, Chen Y-H, Li X-L, Chen F, Huang J-A, et al. Cardiovascular safety, long-term noncardiovascular safety, and efficacy of sodium-glucose cotransporter 2 inhibitors in patients with type 2 diabetes mellitus: a systematic review and meta-analysis with trial sequential analysis. J Am Heart Assoc. 2018;7:e007165.

39. Zinman B, Wanner C, Lachin JM, Fitchett D, Bluhmki E, Hantel $\mathrm{S}$, et al. Empagliflozin, cardiovascular outcomes, and mortality in type 2 diabetes. N Engl J Med. 2015;373:2117-28.

40. Neal B, Perkovic V, Mahaffey KW, de Zeeuw D, Fulcher G, Erondu N, et al. Canagliflozin and cardiovascular and renal events in type 2 diabetes. N Engl J Med. 2017;377(7):644-57.

41. Fralick M, Schneeweiss S, Patorno E. Risk of diabetic ketoacidosis after initiation of an SGLT2 inhibitor. N Engl J Med. 2017;376:2300-2.

42. Pasternak B, Ueda P, Eliasson B, Svensson A-M, Franzén S, Gudbjörnsdottir $S$, et al. Use of sodium glucose cotransporter 2 inhibitors and risk of major cardiovascular events and heart failure: Scandinavian register based cohort study. BMJ. 2019;366:14772.

43. Peters AL, Buschur EO, Buse JB, Cohan P, Diner JC, Hirsch IB. Euglycemic diabetic ketoacidosis: a potential complication of treatment with sodium-glucose cotransporter 2 inhibition. Diabetes Care. 2015;38:1687-93.

44. Singh AK. Sodium-glucose co-transporter-2 inhibitors and euglycemic ketoacidosis: wisdom of hindsight. Indian J Endocrinol Metab. 2015;19:722-30.

45. Roach P, Skierczynski P. Euglycemic diabetic ketoacidosis in a patient with type 2 diabetes after treatment with empagliflozin. Diabetes Care. 2016;39:e3.

46. Scheen AJ. Cardiovascular effects of new oral glucoselowering agents DPP-4 and SGLT-2 inhibitors. Circ Res. 2018;122:1439-59.

47. Birkeland KI, Jørgensen ME, Carstensen B, Persson F, Gulseth HL, Thuresson M, et al. Cardiovascular mortality and morbidity in patients with type 2 diabetes following initiation of sodiumglucose co-transporter-2 inhibitors versus other glucose-lowering drugs (CVD-REAL Nordic): a multinational observational analysis. Lancet Diabetes Endocrinol. 2017;5(9):709-17.

48. Dave CV, Schneeweiss S, Kim D, Fralick M, Tong A, Patorno E. Sodium-glucose cotransporter-2 inhibitors and the risk for severe urinary tract infections: a population-based cohort study. Ann Intern Med. 2019;171(4):248-56.

49. Puckrin R, Saltiel M-P, Reynier P, Azoulay L, Oriana H, Filion KB. SGLT-2 inhibitors and the risk of infections: a systematic review and meta-analysis of randomised controlled trials. Acta Diabetol. 2018;55(5):503-14.

50. Li D, Wang T, Shen S, Fang Z, Dong Y, Tang H. Urinary tract and genital infections in patients with type 2 diabetes treated with sodium-glucose co-transporter 2 inhibitors: a meta-analysis of randomised controlled trials. Diabetes Obes Metab. 2017;19(3):348-55.

51. Gadzhanova S, Pratt N, Roughead E. Use of SGLT2 inhibitors for diabetes and risk of infection: analysis using general practice records from the NPS MedicineWise MedicineInsight program. Diabetes Res Clin Pract. 2017;130:180-5.

52. Rosenstock J, Seman LJ, Jelaska A, Hantel S, Pinnetti S, Hach $\mathrm{T}$, et al. Efficacy and safety of empagliflozin, a sodium glucose cotransporter 2 (SGLT2) inhibitor, as add-on to metformin in type 2 diabetes with mild hyperglycaemia. Diabetes Obes Metab. 2013;15:1154-60. 
53. Rosenstock J, Aggarwal N, Polidori D, Zhao Y, Arbit D, Usiskin $\mathrm{K}$, et al. Dose-ranging effects of canagliflozin, a sodium-glucose cotransporter 2 inhibitor, as add-on to metformin in subjects with type 2 diabetes. Diabetes Care. 2012;35:1232-8.

54. Han SJ, Ha KH, Lee N, Kim DJ. Effectiveness and safety of sodium-glucose co-transporter-2 inhibitors compared with dipeptidyl peptidase- 4 inhibitors in older adults with type 2 diabetes: a nationwide population-based study. Diabetes Obes Metab. 2021;23(3):682-91.

55. Kosiborod M, Lam CSP, Kohsaka S, Kim DJ, Karasik A, Shaw J, et al. Cardiovascular events associated with SGLT-2 inhibitors versus other glucose-lowering drugs: the CVD-REAL 2 study. $\mathrm{J}$ Am Coll Cardiol. 2018;71(23):2628-39.

56. Persson F, Nyström T, Jørgensen ME, Carstensen B, Gulseth HL, Thuresson M, et al. Dapagliflozin is associated with lower risk of cardiovascular events and all-cause mortality in people with type 2 diabetes (CVD-REAL Nordic) when compared with dipeptidyl peptidase-4 inhibitor therapy: a multinational observational study. Diabetes Obes Metab. 2018;20:344-51.

57. Udell JA, Yuan Z, Rush T, Sicignano NM, Galitz M, Rosenthal N. Cardiovascular outcomes and risks after initiation of a sodium glucose cotransporter 2 inhibitor: results from the EASEL population-based cohort study (evidence for cardiovascular outcomes with sodium glucose cotransporter 2 inhibitors in the real world). Circulation. 2018;137:1450-9.

58. Cahn A, Melzer-Cohen C, Pollack R, Chodick G, Shalev V. Acute renal outcomes with sodium-glucose co-transporter-2 inhibitors: real-world data analysis. Diabetes Obes Metab. 2019;21:340-8.
59. Kim YG, Han SJ, Kim DJ, Lee KW, Kim HJ. Association between sodium glucose co-transporter 2 inhibitors and a reduced risk of heart failure in patients with type 2 diabetes mellitus: a real-world nationwide population-based cohort study. Cardiovasc Diabetol. 2018;17:91.

60. Gautam S, Agiro A, Barron J, Power T, Weisman H, White J. Heart failure hospitalisation risk associated with use of two classes of oral antidiabetic medications: an observational, realworld analysis. Cardiovasc Diabetol. 2017;16:93.

61. Zheng SL, Roddick AJ, Aghar-Jaffar R, Shun-Shin MJ, Francis $\mathrm{D}$, Oliver $\mathrm{N}$, et al. Association between use of sodium-glucose cotransporter 2 inhibitors, glucagon-like peptide 1 agonists, and dipeptidyl peptidase 4 inhibitors with all-cause mortality in patients with type 2 diabetes: a systematic review and metaanalysis. J Am Med Assoc. 2018;319:1580-91.

62. Heald A, Davies M, Stedman M, Livingston M, Lunt M, Fryer A, et al. Analysis of English general practice level data linking medication levels, service activity and demography to levels of glycaemic control being achieved in type 2 diabetes to improve clinical practice and patient outcomes. BMJ Open. 2019;9:e28278.

63. Rosenbaum PR, Rubin DB. The central role of the propensity score in observational studies for causal effects. Biometrika. 1983;70(1):41-55.

Publisher's note Springer Nature remains neutral with regard to jurisdictional claims in published maps and institutional affiliations. 